\title{
Dynamics of a Delayed Model for the Transmission of Malicious Objects in Computer Network
}

\author{
Zizhen Zhang ${ }^{1,2}$ and Huizhong Yang ${ }^{2}$ \\ ${ }^{1}$ School of Management Science and Engineering, Anhui University of Finance and Economics, Bengbu 233030, China \\ ${ }^{2}$ Key Laboratory of Advanced Process Control for Light Industry (Ministry of Education), Jiangnan University, Wuxi 214122, China
}

Correspondence should be addressed to Huizhong Yang; yhz@jiangnan.edu.cn

Received 26 June 2014; Accepted 3 July 2014; Published 23 July 2014

Academic Editor: Luca Guerrini

Copyright (c) 2014 Z. Zhang and H. Yang. This is an open access article distributed under the Creative Commons Attribution License, which permits unrestricted use, distribution, and reproduction in any medium, provided the original work is properly cited.

An SEIQRS model for the transmission of malicious objects in computer network with two delays is investigated in this paper. We show that possible combination of the two delays can affect the stability of the model and make the model bifurcate periodic solutions under some certain conditions. For further investigation, properties of the periodic solutions are studied by using the normal form method and center manifold theory. Finally, some numerical simulations are given to justify the theoretical results.

\section{Introduction}

Computer viruses in network have posed a major threat to our work and life with the rapid popularization of the Internet. Many virus propagation models [1-4] have been proposed to understand the way that computer viruses propagate after Kephart and White [5] proposed the first epidemiological model of computer viruses. In [1], Thommes and Coates proposed a modified version of the SEI model to predict the virus propagation in a network. In [3], Wen and Zhong studied an SIR model on bipartite networks and they proved the existence and the asymptotic stability of the endemic equilibrium by applying the theory of the multigroup model. In [4], Mishra and Jha proposed the following SEIQRS model to describe the transmission of malicious objects in computer network by introducing a new compartment quarantine into the SEIRS model proposed in [2]:

$$
\begin{aligned}
& \frac{d S(t)}{d t}=A-\beta S(t) I(t)-d S(t)+\eta R(t), \\
& \frac{d E(t)}{d t}=\beta S(t) I(t)-(d+\mu) E(t), \\
& \frac{d I(t)}{d t}=\mu E(t)-(d+\alpha+\gamma+\delta) I(t),
\end{aligned}
$$

$$
\begin{aligned}
& \frac{d Q(t)}{d t}=\delta I(t)-(d+\alpha+\varepsilon) Q(t), \\
& \frac{d R(t)}{d t}=\gamma I(t)+\varepsilon Q(t)-(d+\eta) R(t),
\end{aligned}
$$

where $S(t), E(t), I(t), Q(t)$, and $R(t)$ denote the sizes of nodes in the states susceptible, exposed, infectious, quarantined, and recovered at time $t$, respectively. $A$ is the rate at which new computers are attached to the network. $d$ is the rate at which computers are disconnected to the network. $\alpha$ is the crashing rate of computers due to the attack of malicious objects. $\beta$ is the transmission rate. $\mu, \gamma, \delta, \varepsilon$, and $\eta$ are the state transition rates.

As is known, an infected computer becomes a recovered one by using antimalicious software and the recovered computer has a temporary immunity, and computer virus models with delay have been studied by many scholars [612]. In [6], Ren et al. investigated local and global stability of a delayed viral infection model in computer virus propagation model. In [8], Dong et al. proposed a delayed SEIR computer virus model and studied the problem of Hopf bifurcation of the model by regarding the delay as a bifurcating parameter. Motivated by the work above, Liu [12] incorporated the time 
delay due to the temporary immunity period into system (1) and proposed the following SEIQRS model with time delay:

$$
\begin{aligned}
& \frac{d S(t)}{d t}=A-\beta S(t) I(t)-d S(t)+\eta R(t-\tau), \\
& \frac{d E(t)}{d t}=\beta S(t) I(t)-(d+\mu) E(t), \\
& \frac{d I(t)}{d t}=\mu E(t)-(d+\alpha+\gamma+\delta) I(t), \\
& \frac{d Q(t)}{d t}=\delta I(t)-(d+\alpha+\varepsilon) Q(t), \\
& \frac{d R(t)}{d t}=\gamma I(t)+\varepsilon Q(t)-d R(t)-\eta R(t-\tau),
\end{aligned}
$$

where $\tau \geq 0$ is the time delay due to the temporary immunity period. However, we know that an infected computer needs a period to clean viruses by antivirus software and then becomes a recovered one. Therefore, there is a time delay before the infected computers develop themselves into the recovered ones. And there have been some papers that deal with the research of Hopf bifurcation of dynamical system with multiple delays [13-18]. In [13], $\mathrm{Xu}$ and He considered a two-neuron network with resonant bilinear terms and two delays. They studied the problem of Hopf bifurcation by regarding the sum of the two delays as a bifurcation parameter. In [16], Meng et al. studied the Hopf bifurcation of a three-species system with two delays by regarding possible combination of the two delays as a bifurcation parameter. Motivated by the work above, we consider the following SEIQRS computer virus model with two delays in the present paper:

$$
\begin{aligned}
& \frac{d S(t)}{d t}=A-\beta S(t) I(t)-d S(t)+\eta R\left(t-\tau_{1}\right), \\
& \frac{d E(t)}{d t}=\beta S(t) I(t)-(d+\mu) E(t), \\
& \frac{d I(t)}{d t}=\mu E(t)-(d+\alpha+\delta) I(t)-\gamma I\left(t-\tau_{2}\right), \\
& \frac{d Q(t)}{d t}=\delta I(t)-(d+\alpha) Q(t)-\varepsilon Q\left(t-\tau_{2}\right), \\
& \frac{d R(t)}{d t}=\gamma I\left(t-\tau_{2}\right)+\varepsilon Q\left(t-\tau_{2}\right)-d R(t)-\eta R\left(t-\tau_{1}\right),
\end{aligned}
$$

where $\tau_{1}$ is the time delay due to the temporary immunity period and $\tau_{2}$ is the time delay due to the period that the infected computer uses to clean viruses by antivirus software.

The main purpose of this paper is to investigate the effects of the two delays on system (3) and the remainder of this paper is organized as follows. Sufficient conditions for local stability and existence of local Hopf bifurcation are obtained by analyzing the distribution of the roots of the associated characteristic equation in Section 2. Properties of the Hopf bifurcation are further investigated by using the normal form method and center manifold theory in Section 3. In Section 4, we give a numerical example to support the theoretical results in the paper.

\section{Local Stability and Existence of Local Hopf Bifurcation}

By a simple computation, it is easy to get that if $R_{0}=$ $A \mu \beta / d(d+\mu)(\alpha+\delta+\gamma+d)>1$, then system (3) has a unique positive equilibrium $P^{*}\left(S^{*}, E^{*}, I^{*}, Q^{*}, R^{*}\right)$, where

$$
\begin{gathered}
S^{*}=\frac{(d+\mu)(d+\alpha+\delta+\gamma)}{\mu \beta}, \quad E^{*}=\frac{(d+\alpha+\delta+\gamma) I^{*}}{\mu}, \\
I^{*}=\frac{(d+\eta)(d+\alpha+\varepsilon)(A \mu \beta-d(d+\mu)(d+\alpha+\delta+\gamma))}{\beta}, \\
Q^{*}=\frac{\delta I^{*}}{d+\alpha+\varepsilon}, \quad R^{*}=\frac{\gamma I^{*}}{d+\eta}+\frac{\varepsilon \delta I^{*}}{(d+\eta)(d+\alpha+\varepsilon)},
\end{gathered}
$$

and $R_{0}$ is the basic reproduction number. It is easy to get the linearization of system (3) at $P^{*}\left(S^{*}, E^{*}, I^{*}, Q^{*}, R^{*}\right)$ :

$$
\begin{aligned}
\frac{d S(t)}{d t}= & a_{11} S(t)+a_{13} I(t)+b_{15} R\left(t-\tau_{1}\right), \\
\frac{d E(t)}{d t}= & a_{21} S(t)+a_{22} E(t)+a_{23} I(t), \\
\frac{d I(t)}{d t}= & a_{32} E(t)+a_{33} I(t)+c_{33} I\left(t-\tau_{2}\right), \\
\frac{d Q(t)}{d t}= & a_{43} I(t)+a_{44} Q(t)+c_{44} Q\left(t-\tau_{2}\right), \\
\frac{d R(t)}{d t}= & a_{55} R(t)+b_{55} R\left(t-\tau_{1}\right)+c_{53} I\left(t-\tau_{2}\right) \\
& +c_{54} Q\left(t-\tau_{2}\right),
\end{aligned}
$$

where

$$
\begin{gathered}
a_{11}=-\left(\beta I^{*}+d\right), \quad a_{13}=-\beta S^{*}, \quad a_{21}=\beta I^{*}, \\
a_{22}=-(d+\mu), \quad a_{23}=\beta S^{*}, \quad a_{32}=\mu, \\
a_{33}=-(d+\alpha+\delta), \quad a_{43}=\delta, \quad a_{44}=-(d+\alpha), \\
a_{55}=-d, \quad b_{15}=\eta, \quad b_{55}=-\eta, \quad c_{33}=-\gamma, \\
c_{44}=-\varepsilon, \quad c_{53}=\gamma, \quad c_{54}=\varepsilon .
\end{gathered}
$$

Thus, the characteristic equation of system (5) is

$$
\begin{aligned}
\lambda^{5} & +m_{4} \lambda^{4}+m_{3} \lambda^{3}+m_{2} \lambda^{2}+m_{1} \lambda+m_{0} \\
& +\left(n_{4} \lambda^{4}+n_{3} \lambda^{3}+n_{2} \lambda^{2}+n_{1} \lambda+n_{0}\right) e^{-\lambda \tau_{1}} \\
& +\left(p_{4} \lambda^{4}+p_{3} \lambda^{3}+p_{2} \lambda^{2}+p_{1} \lambda+p_{0}\right) e^{-\lambda \tau_{2}} \\
& +\left(q_{3} \lambda^{3}+q_{2} \lambda^{2}+q_{1} \lambda+q_{0}\right) e^{-2 \lambda \tau_{2}} \\
& +\left(r_{3} \lambda^{3}+r_{2} \lambda^{2}+r_{1} \lambda+r_{0}\right) e^{-\lambda\left(\tau_{1}+\tau_{2}\right)} \\
& +\left(s_{2} \lambda^{2}+s_{1} \lambda+s_{0}\right) e^{-\lambda\left(\tau_{1}+2 \tau_{2}\right)}=0
\end{aligned}
$$


where

$$
\begin{aligned}
& m_{0}=\left(a_{11} a_{23} a_{32}-a_{11} a_{22} a_{33}-a_{13} a_{21} a_{32}\right) a_{44} a_{55}, \\
& m_{1}=\left(a_{44}+a_{55}\right)\left(a_{13} a_{21} a_{32}-a_{11} a_{23} a_{32}\right)-a_{23} a_{32} a_{44} a_{55} \\
& +a_{11} a_{22} a_{33} a_{44}+a_{11} a_{22} a_{55}\left(a_{33}+a_{44}\right) \\
& +a_{33} a_{44} a_{55}\left(a_{11}+a_{22}\right) \text {, } \\
& m_{2}=a_{23} a_{32}\left(a_{11}+a_{44}+a_{55}\right)-a_{55}\left(a_{11} a_{22}+a_{33} a_{44}\right) \\
& -a_{13} a_{21} a_{32}-a_{11} a_{22}\left(a_{33}+a_{44}\right)-a_{33} a_{44}\left(a_{11}+a_{22}\right) \\
& -a_{55}\left(a_{11}+a_{22}\right)\left(a_{33}+a_{44}\right) \text {, } \\
& m_{3}=a_{11} a_{22}+a_{33} a_{44}+\left(a_{11}+a_{22}\right)\left(a_{33}+a_{44}\right)-a_{23} a_{32} \\
& +a_{55}\left(a_{11}+a_{22}+a_{33}+a_{44}\right) \\
& m_{4}=-\left(a_{11}+a_{22}+a_{33}+a_{44}+a_{55}\right), \\
& n_{0}=\left(a_{11} a_{23} a_{32}-a_{11} a_{22} a_{33}-a_{13} a_{21} a_{32}\right) a_{44} b_{55} \text {, } \\
& n_{1}=a_{11} a_{22} b_{55}\left(a_{33}+a_{44}\right)+a_{33} a_{44} b_{55}\left(a_{11}+a_{22}\right) \\
& +a_{13} a_{21} a_{32} b_{55}-a_{23} a_{32} b_{55}\left(a_{11}+a_{44}\right), \\
& n_{2}=\left(a_{23} a_{32}-a_{11} a_{22}-a_{33} a_{44}\right) b_{55} \\
& -\left(a_{11}+a_{22}\right)\left(a_{33}+a_{44}\right) b_{55} \\
& n_{3}=b_{55}\left(a_{11}+a_{22}+a_{33}+a_{44}\right), \quad n_{4}=-b_{55} \text {, } \\
& p_{0}=\left(a_{11} a_{23}-a_{13} a_{21}\right) a_{32} a_{55} c_{44}-\left(a_{44} c_{33}+a_{33} c_{44}\right) a_{11} a_{22} a_{55} \text {, } \\
& p_{1}=a_{11} a_{22} c_{33}\left(a_{44}+a_{55}\right)+a_{44} a_{55} c_{33}\left(a_{11}+a_{22}\right) \\
& +a_{11} a_{22} c_{44}\left(a_{33}+a_{55}\right)-a_{23} a_{32} c_{44}\left(a_{11}+a_{55}\right) \\
& +a_{33} a_{55} c_{44}\left(a_{11}+a_{22}\right)-a_{13} a_{21} a_{32} c_{44}, \\
& p_{2}=a_{23} a_{32} c_{44}-c_{33}\left(a_{11}+a_{22}\right)\left(a_{44}+a_{55}\right) \\
& -c_{33}\left(a_{11} a_{22}+a_{44} a_{55}\right)-c_{44}\left(a_{11}+a_{22}\right)\left(a_{33}+a_{55}\right) \\
& -c_{44}\left(a_{11} a_{22}+a_{33} a_{55}\right) \text {, } \\
& p_{3}=c_{33}\left(a_{11}+a_{22}+a_{44}+a_{55}\right)+c_{44}\left(a_{11}+a_{22}+a_{33}+a_{55}\right) \text {, } \\
& p_{4}=-\left(c_{33}+c_{44}\right), \quad q_{0}=a_{11} a_{22} a_{55} c_{33} c_{44}, \quad q_{3}=c_{33} c_{44}, \\
& q_{1}=\left(a_{11} a_{22}+a_{11} a_{55}+a_{22} a_{55}\right) c_{33} c_{44}, \\
& q_{2}=-\left(a_{11}+a_{22}+a_{55}\right) c_{33} c_{44} \text {, } \\
& r_{0}=a_{21} a_{32} b_{15}\left(a_{44} c_{53}-a_{43} c_{54}\right)-a_{11} a_{22} b_{55}\left(a_{33} c_{44}+a_{44} c_{33}\right) \\
& -a_{32} b_{55} c_{44}\left(a_{11} a_{23}+a_{13} a_{21}\right) \text {, } \\
& r_{1}=\left(a_{11} a_{22}+a_{11} a_{44}+a_{22} a_{44}\right) b_{55} c_{33}-a_{21} c_{32} b_{15} c_{53} \\
& +\left(a_{11} a_{22}+a_{11} a_{33}+a_{22} a_{33}\right) b_{55} c_{44}+a_{23} a_{32} b_{55} c_{44},
\end{aligned}
$$

$r_{2}=-\left(a_{11}+a_{22}+a_{33}\right) b_{55} c_{44}-\left(a_{11}+a_{22}+a_{44}\right) b_{55} c_{33}$,

$r_{3}=\left(c_{33}+c_{44}\right) b_{55}, \quad s_{0}=a_{21} a_{32} b_{15} c_{44} c_{53}-a_{11} a_{22} b_{55} c_{33} c_{44}$,

$s_{1}=\left(a_{11}+a_{22}\right) b_{55} c_{33} c_{44}, \quad s_{2}=-b_{55} c_{33} c_{44}$.

Case $1\left(\tau_{1}=\tau_{2}=0\right)$. When $\tau_{1}=\tau_{2}=0$, (7) becomes

$$
\begin{aligned}
& \lambda^{5}+A_{14} \lambda^{4}+A_{13} \lambda^{3}+A_{12} \lambda^{2}+A_{11} \lambda+A_{10}=0, \\
& A_{10}=m_{0}+n_{0}+p_{0}+q_{0}+r_{0}+s_{0}, \\
& A_{11}=m_{1}+n_{1}+p_{1}+q_{1}+r_{1}+s_{1}, \\
& A_{12}=m_{2}+n_{2}+p_{2}+q_{2}+r_{2}+s_{2}, \\
& A_{13}=m_{3}+n_{3}+p_{3}+q_{3}+r_{3}, \\
& A_{14}=m_{4}+n_{4}+p_{4} \\
&=\beta I^{*}+5 d+2 \alpha+\mu+\delta+\varepsilon+\gamma+\eta>0 .
\end{aligned}
$$

Let $\operatorname{det}_{1}=A_{14}$. Obviously, $\operatorname{det}_{1}>0$. Therefore, if the condition $\left(H_{1}\right)$ : (10) holds, then the positive equilibrium $P^{*}\left(S^{*}\right.$, $E^{*}, I^{*}, Q^{*}, R^{*}$ ) of system (3) is locally asymptotically stable without delay. Consider

$$
\begin{aligned}
\operatorname{det}_{2} & =\left|\begin{array}{cc}
A_{14} & 1 \\
A_{12} & A_{13}
\end{array}\right|>0, \\
\operatorname{det}_{3} & =\left|\begin{array}{ccc}
A_{14} & 1 & 0 \\
A_{12} & A_{13} & A_{14} \\
0 & A_{11} & A_{12}
\end{array}\right|>0, \\
\operatorname{det}_{4} & =\left|\begin{array}{cccc}
A_{14} & 1 & 0 & 0 \\
A_{12} & A_{13} & A_{14} & 1 \\
A_{10} & A_{11} & A_{12} & A_{13} \\
0 & 0 & A_{10} & A_{11}
\end{array}\right|>0, \\
\operatorname{det}_{5} & =\left|\begin{array}{ccccc}
A_{14} & 1 & 0 & 0 & 0 \\
A_{12} & A_{13} & A_{14} & 1 & 0 \\
A_{10} & A_{11} & A_{12} & A_{13} & A_{14} \\
0 & 0 & A_{10} & A_{11} & A_{12} \\
0 & 0 & 0 & 0 & A_{10}
\end{array}\right|>0 .
\end{aligned}
$$

Case $2\left(\tau_{1}>0, \tau_{2}=0\right)$. When $\tau_{2}=0$, (7) becomes the following form:

$$
\begin{aligned}
\lambda^{5} & +A_{24} \lambda^{4}+A_{23} \lambda^{3}+A_{22} \lambda^{2}+A_{21} \lambda+A_{20} \\
& +\left(B_{24} \lambda^{4}+B_{23} \lambda^{3}+B_{22} \lambda^{2}+B_{21} \lambda+B_{20}\right) e^{-\lambda \tau_{1}}=0
\end{aligned}
$$


where

$$
\begin{gathered}
A_{20}=m_{0}+p_{0}+q_{0}, \quad A_{21}=m_{1}+p_{1}+q_{1}, \\
A_{22}=m_{2}+p_{2}+q_{2}, \quad A_{23}=m_{3}+p_{3}+q_{3}, \\
A_{24}=m_{4}+p_{4}, \quad B_{20}=n_{0}+r_{0}+s_{0}, \\
B_{21}=n_{1}+r_{1}+s_{1}, \quad B_{22}=n_{2}+r_{2}+s_{2}, \\
B_{23}=n_{3}+r_{3}, \quad B_{24}=n_{4} .
\end{gathered}
$$

Let $\lambda=i \omega_{1}\left(\omega_{1}>0\right)$ be a root of (11). Then, we obtain

$$
\begin{aligned}
& \left(B_{21} \omega_{1}-B_{23} \omega_{1}^{3}\right) \sin \tau_{1} \omega_{1}+\left(B_{24} \omega_{1}^{4}-B_{22} \omega_{1}^{2}+B_{20}\right) \cos \tau_{1} \omega_{1} \\
& \quad=A_{22} \omega_{1}^{2}-A_{24} \omega_{1}^{4}-A_{20} \\
& \left(B_{21} \omega_{1}-B_{23} \omega_{1}^{3}\right) \cos \tau_{1} \omega_{1}-\left(B_{24} \omega_{1}^{4}-B_{22} \omega_{1}^{2}+B_{20}\right) \sin \tau_{1} \omega_{1} \\
& \quad=-\omega_{1}^{5}+A_{23} \omega_{1}^{3}-A_{21} \omega_{1} .
\end{aligned}
$$

It follows that

$$
\omega_{1}^{10}+c_{24} \omega_{1}^{8}+c_{23} \omega_{1}^{6}+c_{22} \omega_{1}^{4}+c_{21} \omega_{1}^{2}+c_{20}=0
$$

with

$$
\begin{aligned}
& c_{20}=A_{20}^{2}-B_{20}^{2}, \\
& c_{21}=A_{21}^{2}-B_{21}^{2}-2 A_{20} A_{22}+2 B_{20} B_{22}, \\
& c_{22}=A_{22}^{2}-B_{22}^{2}+2 A_{20} A_{24}-2 A_{21} A_{23}-2 B_{20} B_{24}+2 B_{21} B_{23}, \\
& c_{23}=A_{23}^{2}+2 A_{21}-2 A_{22} A_{24}-B_{23}^{2}+2 B_{22} B_{24}, \\
& c_{24}=A_{24}^{2}-B_{24}^{2}-2 A_{23} .
\end{aligned}
$$

Let $\omega_{1}^{2}=v_{1}$, then (14) becomes

$$
v_{1}^{5}+c_{24} v_{1}^{4}+c_{23} v_{1}^{3}+c_{22} v_{1}^{2}+c_{21} v_{1}+c_{20}=0
$$

If all the parameters of system (3) are given, one can get all the roots of (16) by the software package Matlab. In order to give the main results in this paper, we make the following assumption.

$\left(H_{21}\right)(16)$ has at least one positive real root.

If the condition $\left(H_{21}\right)$ holds, then there exists a $v_{10}$ such that (11) has a pair of purely imaginary roots $\pm i \omega_{10}= \pm i \sqrt{v_{10}}$. For $\omega_{10}$, the corresponding critical value of time delay is

$$
\tau_{10}=\frac{1}{\omega_{10}} \arccos \frac{p_{28} \omega_{10}^{8}+p_{26} \omega_{10}^{6}+p_{24} \omega_{10}^{4}+p_{22} \omega_{10}^{2}+p_{20}}{q_{28} \omega_{10}^{8}+q_{26} \omega_{10}^{6}+q_{24} \omega_{10}^{4}+q_{22} \omega_{10}^{2}+q_{20}},
$$

where

$$
\begin{aligned}
& p_{20}=-A_{20} B_{20}, \quad p_{22}=A_{20} B_{22}-A_{21} B_{21}+A_{22} B_{20}, \\
& p_{24}=A_{21} B_{23}+A_{23} B_{21}-A_{20} B_{24}-A_{22} B_{22}-A_{24} B_{20}, \\
& p_{26}=A_{22} B_{24}+A_{24} B_{22}-A_{23} B_{23}-B_{21}, \\
& p_{28}=B_{23}-A_{24} B_{24}, \quad q_{20}=B_{20}^{2}, \\
& q_{22}=B_{21}^{2}-2 B_{20} B_{22}, \quad q_{24}=B_{22}^{2}+2 B_{20} B_{24}-2 B_{21} B_{23}, \\
& q_{26}=B_{23}^{2}-2 B_{22} B_{24}, \quad q_{28}=B_{24}^{2} .
\end{aligned}
$$

Taking the derivative of $\lambda$ with respect to $\tau_{1}$ in (11), one can obtain

$$
\begin{aligned}
{\left[\frac{d \lambda}{d \tau_{1}}\right]^{-1}=} & -\frac{5 \lambda^{4}+4 A_{24} \lambda^{3}+3 A_{23} \lambda^{2}+2 A_{22} \lambda+A_{21}}{\lambda\left(\lambda^{5}+A_{24} \lambda^{4}+A_{23} \lambda^{3}+A_{22} \lambda^{2}+A_{21} \lambda+A_{20}\right)} \\
& +\frac{4 B_{24} \lambda^{3}+3 B_{23} \lambda^{2}+2 B_{22} \lambda+B_{21}}{\lambda\left(B_{24} \lambda^{4}+B_{23} \lambda^{3}+B_{22} \lambda^{2}+B_{21} \lambda+B_{20}\right)}-\frac{\tau_{1}}{\lambda}
\end{aligned}
$$

Thus,

$$
\begin{aligned}
\operatorname{Re} & {\left[\frac{d \lambda}{d \tau_{1}}\right]_{\lambda=i \omega_{10}}^{-1} } \\
& =\frac{f_{1}^{\prime}\left(v_{1 *}\right)}{\left(B_{21} \omega_{10}-B_{23} \omega_{10}^{3}\right)^{2}+\left(B_{24} \omega_{10}^{4}-B_{22} \omega_{10}^{2}+B_{20}\right)^{2}},
\end{aligned}
$$

where $f_{1}\left(v_{1}\right)=v_{1}^{5}+c_{24} v_{1}^{4}+c_{23} v_{1}^{3}+c_{22} v_{1}^{2}+c_{21} v_{1}+c_{20}$ and $v_{1 *}=\omega_{10}^{2}$.

Obviously, if the condition $\left(H_{22}\right) f_{1}^{\prime}\left(v_{1 *}\right) \neq 0$ holds, then $\operatorname{Re}\left[d \lambda / d \tau_{1}\right]_{\lambda=i \omega_{10}}^{-1} \neq 0$. According to the Hopf bifurcation theorem in [19], we have the following results for system (3).

Theorem 1. For system (3), if the conditions $\left(H_{21}\right)-\left(H_{22}\right)$ hold, then the positive equilibrium $P^{*}\left(S^{*}, E^{*}, I^{*}, Q^{*}, R^{*}\right)$ of system (3) is asymptotically stable for $\tau_{1} \in\left[0, \tau_{10}\right)$ and system (3) undergoes a Hopf bifurcation at the positive equilibrium $P^{*}\left(S^{*}, E^{*}, I^{*}, Q^{*}, R^{*}\right)$ when $\tau_{1}=\tau_{10}$.

Case $3\left(\tau_{1}=0, \tau_{2}>0\right)$. When $\tau_{1}=0$, (7) becomes

$$
\begin{aligned}
\lambda^{5} & +A_{34} \lambda^{4}+A_{33} \lambda^{3}+A_{32} \lambda^{2}+A_{31} \lambda+A_{30} \\
& +\left(B_{34} \lambda^{4}+B_{33} \lambda^{3}+B_{32} \lambda^{2}+B_{31} \lambda+B_{20}\right) e^{-\lambda \tau_{2}} \\
& +\left(C_{33} \lambda^{3}+C_{32} \lambda^{2}+C_{30}\right) e^{-2 \lambda \tau_{2}}=0
\end{aligned}
$$


where

$$
\begin{gathered}
A_{30}=m_{0}+n_{0}, \quad A_{31}=m_{1}+n_{1}, \quad A_{32}=m_{2}+n_{2}, \\
A_{33}=m_{3}+n_{3}, \quad A_{34}=m_{4}+n_{4}, \quad B_{30}=p_{0}+r_{0}, \\
B_{31}=p_{1}+r_{1}, \quad B_{32}=p_{2}+r_{2}, \quad B_{33}=p_{3}+r_{3}, \\
B_{34}=p_{4}, \quad C_{30}=q_{0}+s_{0}, \quad C_{31}=q_{1}+s_{1}, \\
C_{32}=q_{2}+s_{2}, \quad C_{33}=q_{3} .
\end{gathered}
$$

Multiplying $e^{\lambda \tau_{2}}$ on both sides of (21), we have

$$
\begin{aligned}
& B_{34} \lambda^{4}+B_{33} \lambda^{3}+B_{32} \lambda^{2}+B_{31} \lambda+B_{20} \\
& \quad+\left(\lambda^{5}+A_{34} \lambda^{4}+A_{33} \lambda^{3}+A_{32} \lambda^{2}+A_{31} \lambda+A_{30}\right) e^{\lambda \tau_{2}} \\
& \quad+\left(C_{33} \lambda^{3}+C_{32} \lambda^{2}+C_{30}\right) e^{-\lambda \tau_{2}}=0
\end{aligned}
$$

Let $\lambda=i \omega_{2}\left(\omega_{2}>0\right)$ be the root of $(23)$, then we obtain

$$
\begin{aligned}
& M_{31} \sin \tau_{2} \omega_{2}+M_{32} \cos \tau_{2} \omega_{2}=M_{33}, \\
& M_{34} \cos \tau_{2} \omega_{2}-M_{35} \sin \tau_{2} \omega_{2}=M_{36}
\end{aligned}
$$

where

$$
\begin{aligned}
& M_{31}=A_{34} \omega_{2}^{4}+\left(C_{32}-A_{32}\right) \omega_{2}^{2}+A_{30}-C_{30}, \\
& M_{32}=\omega_{2}^{5}-\left(A_{33}+C_{33}\right) \omega_{2}^{3}+\left(A_{31}+C_{31}\right) \omega_{2}, \\
& M_{33}=B_{33} \omega_{2}^{3}-B_{31} \omega_{2}, \\
& M_{34}=A_{34} \omega_{2}^{4}-\left(A_{32}+C_{32}\right) \omega_{2}^{2}+A_{30}+C_{30}, \\
& M_{35}=\omega_{2}^{5}-\left(A_{33}-C_{33}\right) \omega_{2}^{3}+\left(A_{31}-C_{31}\right) \omega_{2}, \\
& M_{36}=B_{32} \omega_{2}^{2}-B_{34} \omega_{2}^{4}-B_{30} .
\end{aligned}
$$

Then, we obtain

$$
\begin{aligned}
& \cos \tau_{2} \omega_{2}=\frac{p_{38} \omega_{2}^{8}+p_{36} \omega_{2}^{6}+p_{34} \omega_{2}^{4}+p_{32} \omega_{2}^{2}+p_{30}}{\omega_{2}^{10}+q_{38} \omega_{2}^{8}+q_{36} \omega_{2}^{6}+q_{34} \omega_{2}^{4}+q_{32} \omega_{2}^{2}+q_{30}} \\
& \sin \tau_{2} \omega_{2}=\frac{p_{37} \omega_{2}^{7}+p_{35} \omega_{2}^{5}+p_{33} \omega_{2}^{3}+p_{31} \omega_{2}}{\omega_{2}^{10}+q_{38} \omega_{2}^{8}+q_{36} \omega_{2}^{6}+q_{34} \omega_{2}^{4}+q_{32} \omega_{2}^{2}+q_{30}}
\end{aligned}
$$

where

$$
\begin{aligned}
& p_{30}= A_{30}\left(C_{30}-B_{30}\right), \\
& p_{31}=\left(A_{31}+C_{31}\right) B_{30}-\left(A_{30}+C_{30}\right) B_{31}, \\
& p_{32}= B_{31}\left(C_{31}-A_{31}\right)+A_{32} B_{30}+A_{30} B_{32}-B_{30} C_{32} \\
&-B_{32} C_{30}, \\
& p_{33}= B_{31}\left(A_{32}+C_{32}\right)+B_{33}\left(A_{30}+C_{30}\right)-B_{30}\left(A_{33}+C_{33}\right) \\
&-B_{32}\left(A_{31}+C_{31}\right), \\
& p_{34}= B_{31}\left(A_{33}-C_{33}\right)+B_{32}\left(C_{32}-A_{32}\right)+B_{33}\left(A_{31}-C_{31}\right) \\
&+B_{34}\left(C_{30}-A_{30}\right)-A_{34} B_{30}, \\
& p_{35}= B_{32}\left(A_{33}+C_{33}\right)+B_{34}\left(A_{31}+C_{31}\right)-B_{33}\left(A_{32}+C_{32}\right) \\
&-A_{34} B_{31}, \\
& p_{36}= A_{34} B_{32}+A_{32} B_{34}-A_{33} B_{33}-B_{31}+B_{33} C_{33}-B_{34} C_{32}, \\
& p_{37}= A_{34} B_{33}-B_{32}-\left(A_{33}+C_{33}\right) B_{34}, \\
& p_{38}= B_{33}-A_{34} C_{34}, \\
& q_{30}= A_{30}^{2}-C_{30}^{2}, \quad q_{32}=A_{31}^{2}-C_{31}^{2}-2 A_{30} A_{32}+2 C_{30} C_{32}, \\
& q_{34}= A_{32}^{2}-C_{32}^{2}-2 A_{30} A_{34}+2 C_{31} C_{33}-2 A_{31} A_{33}, \\
& q_{36}= A_{33}^{2}-C_{33}^{2}+2 A_{31}-2 A_{32} A_{34}, \\
& q_{38}=A_{34}^{2}-2 A_{33} .
\end{aligned}
$$

Then, we obtain

$$
\begin{gathered}
\omega_{2}^{20}+c_{39} \omega_{2}^{18}+c_{38} \omega_{2}^{16}+c_{37} \omega_{2}^{14}+c_{36} \omega_{2}^{12}+c_{35} \omega_{2}^{10} \\
+c_{34} \omega_{2}^{8}+c_{33} \omega_{2}^{6}+c_{32} \omega_{2}^{4}+c_{31} \omega_{2}^{2}+c_{30}=0
\end{gathered}
$$

where

$$
\begin{aligned}
c_{30}= & q_{30}^{2}-p_{30}^{2}, \quad c_{31}=2 q_{30} q_{32}-2 p_{30} p_{32}-p_{31}^{2}, \\
c_{32}= & q_{32}^{2}-p_{32}^{2}+2 q_{30} q_{34}-2 p_{30} p_{34}-2 p_{31} p_{33}, \\
c_{33}= & 2 q_{30} q_{36}+2 q_{32} q_{34}-2 p_{30} p_{36}-2 p_{31} p_{35} \\
& -2 p_{32} p_{34}-p_{33}^{2}, \\
c_{34}= & q_{34}^{2}-p_{34}^{2}+2 q_{30} q_{38}+2 q_{32} q_{36}-2 p_{30} p_{38} \\
& -2 p_{31} p_{37}-2 p_{32} p_{36}-2 p_{33} p_{35}, \\
c_{35}= & 2 q_{32} q_{38}-2 p_{32} p_{38}-2 p_{33} p_{37}-2 p_{34} p_{36}-p_{35}^{2}, \\
c_{36}= & q_{36}^{2}-p_{36}^{2}+2 q_{34} q_{38}+2 q_{32}-2 p_{34} p_{38}-2 p_{35} p_{37}, \\
c_{37}= & 2 q_{34}+2 q_{36} q_{38}-p_{36} p_{38}-p_{37}^{2}, \\
c_{38}= & q_{38}^{2}-p_{38}^{2}+2 q_{36}, \quad c_{39}=2 q_{38} .
\end{aligned}
$$


Let $\omega_{2}^{2}=v_{2}$, then (28) becomes

$$
\begin{aligned}
v_{2}^{10} & +c_{39} v_{2}^{9}+c_{38} v_{2}^{8}+c_{37} v_{2}^{7}+c_{36} v_{2}^{6}+c_{35} v_{2}^{5} \\
& +c_{34} v_{2}^{4}+c_{33} v_{2}^{3}+c_{32} v_{2}^{2}+c_{31} v_{2}+c_{30}=0 .
\end{aligned}
$$

Similar as in Case 2, we make the following assumption. $\left(H_{31}\right)(30)$ has at least one positive real root. If the condition $\left(H_{31}\right)$ holds, then there exists a $v_{20}$ such that (23) has a pair of purely imaginary roots $\pm i \omega_{20}= \pm i \sqrt{v_{20}}$. For $\omega_{20}$, the corresponding critical value of time delay is

$\tau_{20}$

$$
=\frac{1}{\omega_{20}} \arccos \frac{p_{38} \omega_{20}^{8}+p_{36} \omega_{20}^{6}+p_{34} \omega_{20}^{4}+p_{32} \omega_{20}^{2}+p_{30}}{\omega_{20}^{10}+q_{38} \omega_{20}^{8}+q_{36} \omega_{20}^{6}+q_{34} \omega_{20}^{4}+q_{32} \omega_{20}^{2}+q_{30}} .
$$

Differentiating two sides of (23) with respect to $\tau_{2}$, we have

$$
\left[\frac{d \lambda}{d \tau_{2}}\right]^{-1}=\frac{g_{31}(\lambda)+g_{32}(\lambda) e^{\lambda \tau_{2}}+g_{33}(\lambda) e^{-\lambda \tau_{2}}}{g_{34}(\lambda) e^{-\lambda \tau_{2}}-g_{35}(\lambda) e^{\lambda \tau_{2}}}-\frac{\tau_{2}}{\lambda},
$$

where

$$
\begin{aligned}
& g_{31}(\lambda)=4 B_{34} \lambda^{3}+3 B_{33} \lambda^{2}+2 B_{32} \lambda+B_{31}, \\
& g_{32}(\lambda)=5 \lambda^{4}+4 A_{34} \lambda^{3}+3 A_{33} \lambda^{2}+2 A_{32} \lambda+A_{31} \\
& g_{33}(\lambda)=3 C_{33} \lambda^{2}+2 C_{32} \lambda+C_{31}, \\
& g_{34}(\lambda)=C_{33} \lambda^{4}+C_{32} \lambda^{3}+C_{31} \lambda^{2}+C_{30} \lambda \\
& g_{35}(\lambda)=\lambda^{6}+A_{34} \lambda^{5}+A_{33} \lambda^{4}+A_{32} \lambda^{3}+A_{31} \lambda^{2}+A_{30} \lambda
\end{aligned}
$$

Thus,

$$
\operatorname{Re}\left[\frac{d \lambda}{d \tau_{2}}\right]_{\lambda=i \omega_{20}}^{-1}=\frac{P_{3 R} Q_{3 R}+P_{3 I} Q_{3 I}}{Q_{3 R}^{2}+Q_{3 I}^{2}}
$$

where

$$
\begin{aligned}
P_{3 R}= & \left(5 \omega_{20}^{4}-3\left(A_{33}+C_{33}\right) 5 \omega_{20}^{2}+A_{31}+C_{31}\right) \cos \tau_{20} \omega_{20} \\
& +\left(4 A_{34} \omega_{20}^{3}+2\left(C_{32}-A_{32}\right) \omega_{20}\right) \sin \tau_{20} \omega_{20}+B_{31} \\
& -3 B_{33} \omega_{20}^{2}, \\
P_{3 I}= & \left(5 \omega_{20}^{4}-3\left(A_{33}-C_{33}\right) 5 \omega_{20}^{2}+A_{31}-C_{31}\right) \sin \tau_{20} \omega_{20} \\
& -\left(4 A_{34} \omega_{20}^{3}-2\left(A_{32}+C_{32}\right) \omega_{20}\right) \cos \tau_{20} \omega_{20}+2 B_{32} \omega_{20} \\
& -4 B_{34} \omega_{20}^{3},
\end{aligned}
$$

$$
\begin{aligned}
Q_{3 R}= & \left.\left(A_{33}+C_{33}\right) \omega_{20}^{4}-\omega_{20}^{6}-\left(A_{31}+C_{31}\right) \omega_{20}^{2}\right) \cos \tau_{20} \omega_{20} \\
& -\left(A_{34} \omega_{20}^{5}+\left(A_{32}-C_{32}\right) \omega_{20}^{3}+\left(A_{30}-C_{30}\right) \omega_{20}\right) \\
& \times \sin \tau_{20} \omega_{20}, \\
Q_{3 I}= & \left.\left(A_{33}-C_{33}\right) \omega_{20}^{4}-\omega_{20}^{6}-\left(A_{31}-C_{31}\right) \omega_{20}^{2}\right) \sin \tau_{20} \omega_{20} \\
& +\left(A_{34} \omega_{20}^{5}-\left(A_{32}+C_{32}\right) \omega_{20}^{3}+\left(A_{30}+C_{30}\right) \omega_{20}\right) \\
& \times \cos \tau_{20} \omega_{20} .
\end{aligned}
$$

Obviously, if the condition $\left(H_{32}\right) P_{3 R} Q_{3 R}+P_{3 I} Q_{3 I} \neq 0$ holds, then $\operatorname{Re}\left[d \lambda / d \tau_{2}\right]_{\lambda=i \omega_{20}}^{-1} \neq 0$. According to the Hopf bifurcation theorem in [19], we have the following results for system (3).

Theorem 2. For system (3), if the conditions $\left(H_{31}\right)-\left(H_{32}\right)$ hold, then the positive equilibrium $P^{*}\left(S^{*}, E^{*}, I^{*}, Q^{*}, R^{*}\right)$ of system (3) is asymptotically stable for $\tau_{2} \in\left[0, \tau_{20}\right)$ and system (3) undergoes a Hopf bifurcation at the positive equilibrium $P^{*}\left(S^{*}, E^{*}, I^{*}, Q^{*}, R^{*}\right)$ when $\tau_{2}=\tau_{20}$.

Case $4\left(\tau_{1}>0, \tau_{2}>0, \tau_{2} \in\left(0, \tau_{20}\right)\right)$. We consider system (3) under the condition that $\tau_{2}$ is in its stable interval and $\tau_{1}$ is a bifurcation parameter.

Let $\lambda=i \omega_{1 *}\left(\omega_{1 *}>0\right)$ be the root of $(7)$, then we obtain

$$
\begin{aligned}
& M_{41} \sin \tau_{1} \omega_{1 *}+M_{42} \cos \tau_{1} \omega_{1 *}=M_{43}, \\
& M_{41} \cos \tau_{1} \omega_{1 *}-M_{42} \sin \tau_{1} \omega_{1 *}=M_{44},
\end{aligned}
$$

where

$$
\begin{aligned}
M_{41}= & n_{1} \omega_{1 *}-n_{3} \omega_{1 *}^{3}+\left(r_{1} \omega_{1 *}-r_{3} \omega_{1 *}^{3}\right) \cos \tau_{2} \omega_{1 *} \\
& -\left(r_{0}-r_{2} \omega_{1 *}^{2}\right) \sin \tau_{2} \omega_{1 *}+s_{1} \omega_{1 *} \cos 2 \tau_{2} \omega_{1 *} \\
& -\left(s_{0}-s_{2} \omega_{1 *}^{2}\right) \sin 2 \tau_{2} \omega_{1 *}, \\
M_{42}= & n_{4} \omega_{1 *}^{4}-n_{2} \omega_{1 *}^{2}+n_{0}+\left(r_{1} \omega_{1 *}-r_{3} \omega_{1 *}^{3}\right) \sin \tau_{2} \omega_{1 *} \\
& +\left(r_{0}-r_{2} \omega_{1 *}^{2}\right) \cos \tau_{2} \omega_{1 *}+s_{1} \omega_{1 *} \sin 2 \tau_{2} \omega_{1 *} \\
& +\left(s_{0}-s_{2} \omega_{1 *}^{2}\right) \cos 2 \tau_{2} \omega_{1 *}, \\
M_{43}= & \left(p_{3} \omega_{1 *}^{3}-p_{1} \omega_{1 *}\right) \sin \tau_{2} \omega_{1 *} \\
& -\left(p_{4} \omega_{1 *}^{4}-p_{2} \omega_{1 *}^{2}+p_{0}\right) \cos \tau_{2} \omega_{1 *} \\
& +\left(q_{3} \omega_{1 *}^{3}-q_{1} \omega_{1 *}\right) \sin 2 \tau_{2} \omega_{1 *} \\
& +\left(q_{2} \omega_{1 *}^{2}-q_{0}\right) \cos \tau_{2} \omega_{1 *}+m_{2} \omega_{1 *}^{2}-m_{4} \omega_{1 *}^{4}-m_{0} \\
= & \left(p_{3} \omega_{1 *}^{3}-p_{1} \omega_{1 *}\right) \cos \tau_{2} \omega_{1 *} \\
& +\left(p_{4} \omega_{1 *}^{4}-p_{2} \omega_{1 *}^{2}+p_{0}\right) \sin \tau_{2} \omega_{1 *} \\
& +\left(q_{3} \omega_{1 *}^{3}-q_{1} \omega_{1 *}\right) \cos 2 \tau_{2} \omega_{1 *} \\
& -\left(q_{2} \omega_{1 *}^{2}-q_{0}\right) \sin \tau_{2} \omega_{1 *}+m_{3} \omega_{1 *}^{3}-\omega_{1 *}^{5}-m_{1} \omega_{1 *} \cdot
\end{aligned}
$$


Then, we can obtain

$$
\begin{aligned}
f_{40}\left(\omega_{1 *}\right)+2 f_{41}\left(\omega_{1 *}\right) \cos \tau_{2} \omega_{1 *}+2 f_{42}\left(\omega_{1 *}\right) \sin \tau_{2} \omega_{1 *} \\
+2 f_{43}\left(\omega_{1 *}\right) \cos 2 \tau_{2} \omega_{1 *}+2 f_{44}\left(\omega_{1 *}\right) \sin 2 \tau_{2} \omega_{1 *} \\
+2 f_{45}\left(\omega_{1 *}\right) \cos \tau_{2} \omega_{1 *} \cos 2 \tau_{2} \omega_{1 *} \\
+2 f_{46}\left(\omega_{1 *}\right) \cos \tau_{2} \omega_{1 *} \sin 2 \tau_{2} \omega_{1 *} \\
+2 f_{47}\left(\omega_{1 *}\right) \sin \tau_{2} \omega_{1 *} \sin 2 \tau_{2} \omega_{1 *} \\
+2 f_{48}\left(\omega_{1 *}\right) \sin \tau_{2} \omega_{1 *} \cos 2 \tau_{2} \omega_{1 *}=0,
\end{aligned}
$$

where

$$
\begin{aligned}
f_{40}\left(\omega_{1 *}\right)=\omega_{1 *}^{10} & +\left(m_{4}^{2}-n_{4}^{2}+p_{4}^{2}-2 m_{3}\right) \omega_{1 *}^{8} \\
+ & \left(m_{3}^{2}-n_{3}^{2}+p_{3}^{2}+q_{3}^{2}-r_{3}^{2}+2 m_{1}-2 m_{2} m_{4}\right. \\
& \left.\quad+2 n_{2} n_{4}-2 p_{2} p_{4}\right) \omega_{1 *}^{6} \\
+ & \left(m_{2}^{2}-n_{2}^{2}+p_{2}^{2}+q_{2}^{2}-r_{2}^{2}-s_{2}^{2}+2 m_{0} m_{4}\right. \\
& \quad-2 m_{1} m_{3}+2 n_{0} n_{4}+2 n_{1} n_{3}-2 p_{1} p_{3}-2 q_{1} q_{3} \\
& \left.+2 r_{1} r_{3}\right) \omega_{1 *}^{4} \\
+ & \left(m_{1}^{2}-n_{1}^{2}+p_{1}^{2}+q_{1}^{2}-r_{1}^{2}-s_{1}^{2}-2 m_{0} m_{2}\right. \\
& +2 n_{0} n_{2}-2 p_{0} p_{2}-2 q_{0} q_{2}+2 r_{0} r_{2} \\
& \left.+2 s_{0} s_{2}\right) \omega_{1 *}^{2}+m_{0}^{2}-n_{0}^{2}+p_{0}^{2}+q_{0}^{2}-r_{0}^{2}-s_{0}^{2},
\end{aligned}
$$$$
f_{41}\left(\omega_{1 *}\right)=\left(m_{4} p_{4}-p_{3}\right) \omega_{1 *}^{8}
$$$$
+\left(m_{3} p_{3}+p_{1}-m_{2} p_{4}-m_{4} p_{2}-n_{3} r_{3}+n_{4} r_{2}\right) \omega_{1 *}^{6}
$$$$
+\left(m_{0} p_{4}-m_{1} p_{3}+m_{2} p_{2}-m_{3} p_{1}+m_{4} p_{0}+n_{1} r_{3}\right.
$$$$
\left.-n_{2} r_{2}+n_{3} r_{1}-n_{4} r_{0}\right) \omega_{1 *}^{4}
$$$$
+\left(m_{1} p_{1}-m_{0} p_{2}-m_{2} p_{0}+n_{0} r_{2}-n_{1} r_{1}\right.
$$$$
\left.+n_{2} r_{0}\right) \omega_{1 *}^{2}+m_{0} p_{0}-n_{0} r_{0}
$$

$$
\begin{aligned}
f_{42}\left(\omega_{1 *}\right)= & -p_{4} \omega_{1 *}^{9}+\left(m_{3} p_{4}-m_{4} p_{3}+n_{4} r_{3}+p_{2}\right) \omega_{1 *}^{7} \\
& +\left(m_{2} p_{3}-m_{1} p_{4}-m_{3} p_{2}+m_{4} p_{1}-n_{2} r_{3}\right. \\
& \left.+n_{3} r_{2}-n_{4} r_{1}-p_{0}\right) \omega_{1 *}^{5} \\
& +\left(m_{1} p_{2}-m_{0} p_{3}-m_{2} p_{1}+m_{3} p_{0}+n_{0} r_{3}\right. \\
& \left.\quad-n_{1} r_{2}+n_{2} r_{1}-n_{3} r_{0}\right) \omega_{1 *}^{3} \\
& +\left(m_{0} p_{1}-m_{1} p_{0}-n_{0} r_{1}+n_{1} r_{0}\right) \omega_{1 *}, \\
f_{43}\left(\omega_{1 *}\right)= & -q_{3} \omega_{1 *}^{8}+\left(m_{3} q_{3}-m_{4} q_{2}+q_{1}\right) \omega_{1 *}^{6} \\
& +\left(m_{2} q_{2}-m_{1} q_{3}-m_{3} q_{1}+m_{4} q_{0}+n_{3} s_{1}\right) \omega_{1 *}^{4} \\
& +\left(m_{1} q_{1}-m_{0} q_{2}-m_{2} q_{0}-n_{1} s_{1}\right) \omega_{1 *}^{2},
\end{aligned}
$$

$$
\begin{aligned}
f_{44}\left(\omega_{1 *}\right)= & q_{2} \omega_{1 *}^{7}+\left(n_{3} s_{2}-m_{3} q_{2}-q_{0}\right) \omega_{1 *}^{5} \\
& +\left(m_{1} q_{2}+m_{3} q_{0}-n_{1} s_{2}-n_{3} s_{0}\right) \omega_{1 *}^{3} \\
& +\left(n_{1} s_{0}-m_{1} q_{0}\right) \omega_{1 *}, \\
f_{45}\left(\omega_{1 *}\right)= & \left(p_{3} q_{3}-p_{4} q_{2}\right) \omega_{1 *}^{6} \\
& +\left(p_{2} q_{2}+p_{4} q_{0}-p_{1} q_{3}-p_{3} q_{1}+r_{2} s_{2}-r_{3} s_{1}\right) \omega_{1 *}^{4} \\
& +\left(p_{1} q_{1}-p_{0} q_{2}-p_{2} q_{0}-r_{0} s_{2}+r_{1} s_{1}-r_{2} s_{0}\right) \omega_{1 *}^{2} \\
& +p_{0} q_{0}+r_{0} s_{0}, \\
f_{46}\left(\omega_{1 *}\right)= & -p_{4} q_{3} \omega_{1 *}^{7}+\left(p_{2} q_{3}-p_{3} q_{2}+p_{4} q_{1}+r_{3} s_{2}\right) \omega_{1 *}^{5} \\
& +\left(p_{1} q_{2}-p_{0} q_{3}-p_{2} q_{1}-p_{3} q_{0}-r_{1} s_{2}\right. \\
& \left.+r_{2} s_{1}-r_{3} s_{0}\right) \omega_{1 *}^{3} \\
& +\left(p_{0} q_{1}-p_{1} q_{0}-r_{1} s_{0}-r_{0} s_{1}\right) \omega_{1 *}, \\
f_{47}\left(\omega_{1 *}\right)= & \left.p_{3} q_{3}-p_{4} q_{2}\right) \omega_{1 *}^{6} \\
& +\left(p_{2} q_{2}-p_{1} q_{3}-p_{3} q_{1}-p_{4} q_{0}-r_{2} s_{2}+r_{3} s_{1}\right) \omega_{1 *}^{4} \\
& +\left(p_{1} q_{1}-p_{0} q_{2}-p_{2} q_{0}+r_{0} s_{2}-r_{1} s_{1}+r_{2} s_{0}\right) \omega_{1 *}^{2} \\
& +p_{0} q_{0}-r_{0} s_{0}, \\
& +\left(p_{1} q_{0}+p_{0} q_{1}+r_{0} s_{1}-r_{1} s_{0}\right) \omega_{1 *} . \\
& +\left(p_{0} q_{3}-p_{1} q_{2}+p_{2} q_{1}-p_{3} q_{0}+r_{1} s_{2}\right. \\
& \left.-r_{2} s_{1}-r_{3} s_{0}\right) \omega_{1 *}^{3} \\
f_{48}\left(\omega_{1 *}\right)= & p_{4} q_{3} \omega_{1 *}^{7}+\left(p_{3} q_{2}-p_{2} q_{3}-p_{4} q_{1}-r_{3} s_{2}\right) \omega_{1 *}^{5} \\
& +p_{1}
\end{aligned}
$$

In order to give the main results in this paper, we make the following assumption.

$\left(H_{41}\right)$ (38) has at least one positive real root. If the conditions $\left(H_{41}\right)$ hold, then there exists a $\omega_{10}^{*}$ such that $(7)$ has a pair of purely imaginary roots $\pm i \omega_{10}^{*}$. For $\omega_{10}^{*}$, the corresponding critical value of time delay is

$$
\tau_{10}^{*}=\left.\frac{1}{\omega_{10}^{*}} \arccos \frac{M_{41} M_{44}+M_{42} M_{43}}{M_{41}^{2}+M_{42}^{2}}\right|_{\omega_{1 *}=\omega_{10}^{*}} .
$$

Differentiating two sides of (7) with respect to $\tau_{1}$, we have

$$
\begin{aligned}
{\left[\frac{d \lambda}{d \tau_{1}}\right]^{-1}=} & \left(g_{41}(\lambda)+g_{42}(\lambda) e^{-\lambda \tau_{1}}+g_{43}(\lambda) e^{-\lambda \tau_{2}}\right. \\
& +g_{44}(\lambda) e^{-\lambda\left(\tau_{1}+\tau_{2}\right)}+g_{45}(\lambda) e^{-2 \lambda \tau_{2}} \\
& \left.+g_{46}(\lambda) e^{-\lambda\left(\tau_{1}+2 \tau_{2}\right)}\right) \\
& \times\left(g_{47}(\lambda) e^{-\lambda \tau_{1}}+g_{48}(\lambda) e^{-\lambda\left(\tau_{1}+\tau_{2}\right)}\right. \\
& \left.+g_{49}(\lambda) e^{-\lambda\left(\tau_{1}+2 \tau_{2}\right)}\right)^{-1} \\
& -\frac{\tau_{1}}{\lambda}
\end{aligned}
$$


where

$$
\begin{aligned}
g_{41}(\lambda)= & 5 \lambda^{4}+4 m_{4} \lambda^{3}+3 m_{3} \lambda^{2}+2 m_{2} \lambda+m_{1}, \\
g_{42}(\lambda)= & 4 n_{4} \lambda^{3}+3 n_{3} \lambda^{2}+2 n_{2} \lambda+n_{1}, \\
g_{43}(\lambda)= & -\tau_{2} p_{4} \lambda^{4}+\left(4 p_{4}-\tau_{2} p_{3}\right) \lambda^{3}+\left(3 p_{3}-\tau_{2} p_{2}\right) \lambda^{2} \\
& +\left(2 p_{2}+\tau_{2} p_{1}\right) \lambda+p_{1}-\tau_{2} p_{0}, \\
g_{44}(\lambda)= & -\tau_{2} q_{3}^{3}+\left(3 q_{3}-\tau_{2} q_{2}\right)^{2}+\left(2 q_{2}-\tau_{2} p_{1}\right) \lambda \\
& +q_{1}-\tau_{2} q_{0}, \\
g_{45}(\lambda)= & -\tau_{2} s_{2} \lambda^{2}+\left(2 s_{2}-\tau_{2} s_{1}\right) \lambda+s_{1}-\tau_{2} s_{0}, \\
g_{46}(\lambda)= & 3 r_{3} \lambda^{2}+2 r_{2} \lambda+r_{1}, \\
g_{47}(\lambda)= & n_{4} \lambda^{5}+n_{3} \lambda^{4}+n_{2} \lambda^{3}+n_{1} \lambda^{2}+n_{0} \lambda, \\
g_{48}(\lambda)= & r_{3} \lambda^{4}+r_{2} \lambda^{3}+r_{1} \lambda^{2}+r_{0} \lambda, \\
g_{49}(\lambda)= & s_{2} \lambda^{3}+s_{1} \lambda^{2}+s_{0} \lambda .
\end{aligned}
$$

Thus,

$$
\operatorname{Re}\left[\frac{d \lambda}{d \tau_{1}}\right]_{\lambda=i \omega_{10}^{*}}^{-1}=\frac{P_{4 R} Q_{4 R}+P_{4 I} Q_{4 I}}{Q_{4 R}^{2}+Q_{4 I}^{2}}
$$

where

$$
\begin{aligned}
& P_{4 R}=5\left(\omega_{10}^{*}\right)^{4}-3 m_{3}\left(\omega_{10}^{*}\right)^{2}+m_{1} \\
& +\left(2 n_{2} \omega_{10}^{*}-4 n_{4}\left(\omega_{10}^{*}\right)^{3}+2 r_{2} \omega_{10}^{*} \cos \tau_{2} \omega_{10}^{*}\right. \\
& +\left(3 r_{3}\left(\omega_{10}^{*}\right)^{2}-r_{1}\right) \sin \tau_{2} \omega_{10}^{*} \\
& +\left(2 s_{2}-\tau_{2} s_{1}\right) \omega_{10}^{*} \cos 2 \tau_{2}^{*} \omega_{10}^{*} \\
& \left.-\left(\tau_{2} s_{2}\left(\omega_{10}^{*}\right)^{2}+s_{1}-\tau_{2} s_{0}\right) \sin 2 \tau_{2} \omega_{10}^{*}\right) \sin \tau_{10}^{*} \omega_{10}^{*} \\
& +\left(n_{1}-3 n_{3}\left(\omega_{10}^{*}\right)^{2}+2 r_{2} \omega_{10}^{*} \sin \tau_{2} \omega_{10}^{*}\right. \\
& +\left(r_{1}-3 r_{3}\left(\omega_{10}^{*}\right)^{2}\right) \cos \tau_{2} \omega_{10}^{*} \\
& +\left(2 s_{2}-\tau_{2} s_{1}\right) \omega_{10}^{*} \sin 2 \tau_{2} \omega_{10}^{*} \\
& \left.+\left(\tau_{2} s_{2}\left(\omega_{10}^{*}\right)^{2}+s_{1}-\tau_{2} s_{0}\right) \cos 2 \tau_{2} \omega_{10}^{*}\right) \cos \tau_{2} \omega_{10}^{*} \\
& +\left(\left(2 p_{2}-\tau_{2} p_{1}\right) \omega_{10}^{*}-\left(4 p_{4}-\tau_{2} p_{3}\right)\left(\omega_{10}^{*}\right)^{3}\right) \sin \tau_{2} \omega_{10}^{*} \\
& +\left(\left(\tau_{2} p_{2}-3 p_{3}\right)\left(\omega_{10}^{*}\right)^{2}-\tau_{2} p_{4}\left(\omega_{10}^{*}\right)^{4}+p_{1}-\tau_{2} p_{0}\right) \\
& \times \cos \tau_{2} \omega_{10}^{*} \\
& +\left(\tau_{2} q_{3}\left(\omega_{10}^{*}\right)^{3}+\left(2 q_{2}-\tau_{2} q_{1}\right) \omega_{10}^{*}\right) \sin 2 \tau_{2} \omega_{10}^{*} \\
& +\left(\left(\tau_{2} q_{2}-3 q_{3}\right)\left(\omega_{10}^{*}\right)^{2}+q_{1}-\tau_{2} q_{0}\right) \cos 2 \tau_{2} \omega_{10}^{*},
\end{aligned}
$$

$$
\begin{aligned}
P_{4 I}=2 m_{2} \omega_{10}^{*}-4 m_{4}\left(\omega_{10}^{*}\right)^{3}+2 r_{2} \omega_{10}^{*} \cos \tau_{2} \omega_{10}^{*} \\
+\left(\left(3 r_{3}\left(\omega_{10}^{*}\right)^{2}-r_{1}\right) \sin \tau_{2} \omega_{10}^{*}\right. \\
\quad+\left(2 s_{2}-\tau_{2} s_{1}\right) \omega_{10}^{*} \cos 2 \tau_{2} \omega_{10}^{*} \\
\left.\quad-\left(\tau_{2} s_{2}\left(\omega_{10}^{*}\right)^{2}+s_{1}-\tau_{2} s_{0}\right) \sin 2 \tau_{2} \omega_{10}^{*}\right) \cos \tau_{2} \omega_{10}^{*} \\
+\left(3 n_{3}\left(\omega_{10}^{*}\right)^{2}-n_{1}-2 r_{2} \omega_{10}^{*} \sin \tau_{2} \omega_{10}^{*}\right. \\
\quad-\left(r_{1}-3 r_{3}\left(\omega_{10}^{*}\right)^{2}\right) \cos \tau_{2} \omega_{10}^{*} \\
\quad-\left(2 s_{2}-\tau_{2} s_{1}\right) \omega_{10}^{*} \sin 2 \tau_{2} \omega_{10}^{*} \\
\left.\quad-\left(\tau_{2} s_{2}\left(\omega_{10}^{*}\right)^{2}+s_{1}-\tau_{2} s_{0}\right) \cos 2 \tau_{2} \omega_{10}^{*}\right) \sin \tau_{10}^{*} \omega_{10}^{*} \\
+\left(\left(2 p_{2}-\tau_{2} p_{1}\right) \omega_{10}^{*}-\left(4 p_{4}-\tau_{2} p_{3}\right)\left(\omega_{10}^{*}\right)^{3}\right) \cos \tau_{2} \omega_{10}^{*} \\
-\left(\left(\tau_{2} p_{2}-3 p_{3}\right)\left(\omega_{10}^{*}\right)^{2}-\tau_{2} p_{4}\left(\omega_{10}^{*}\right)^{4}+p_{1}-\tau_{2} p_{0}\right) \\
\times \sin \tau_{2} \omega_{10}^{*} \\
+\left(\tau_{2} q_{3}\left(\omega_{10}^{*}\right)^{3}+\left(2 q_{2}-\tau_{2} q_{1}\right) \omega_{10}^{*}\right) \cos 2 \tau_{2} \omega_{10}^{*} \\
-\left(\left(\tau_{2} q_{0}-3 q_{3}\right)\left(\omega_{10}^{*}\right)^{2}+q_{1}-\tau_{2} q_{0}\right) \sin 2 \tau_{2} \omega_{10}^{*},
\end{aligned}
$$

$$
\begin{aligned}
& Q_{4 R}=\left(n_{4}\left(\omega_{10}^{*}\right)^{5}-n_{2}\left(\omega_{10}^{*}\right)^{3}+n_{0} \omega_{10}^{*}\right. \\
& +\left(r_{0} \omega_{10}^{*}-r_{2}\left(\omega_{10}^{*}\right)^{3}\right) \cos \tau_{2} \omega_{10}^{*} \\
& -\left(r_{3}\left(\omega_{10}^{*}\right)^{4}-r_{1}\left(\omega_{10}^{*}\right)^{2}\right) \sin \tau_{2} \omega_{10}^{*} \\
& +\left(s_{0} \omega_{10}^{*}-s_{2}\left(\omega_{10}^{*}\right)^{3}\right) \cos \tau_{2} \omega_{10}^{*} \\
& \left.+s_{1}\left(\omega_{10}^{*}\right)^{2} \sin 2 \tau_{2} \omega_{10}^{*}\right) \sin \tau_{10}^{*} \omega_{10}^{*} \\
& +\left(n_{3}\left(\omega_{10}^{*}\right)^{4}-n_{1}\left(\omega_{10}^{*}\right)^{2}+\left(r_{0} \omega_{10}^{*}-r_{2}\left(\omega_{10}^{*}\right)^{3}\right) \sin \tau_{2} \omega_{10}^{*}\right. \\
& +\left(r_{3}\left(\omega_{10}^{*}\right)^{4}-r_{1}\left(\omega_{10}^{*}\right)^{2}\right) \cos \tau_{2} \omega_{10}^{*} \\
& +\left(s_{0} \omega_{10}^{*}-s_{2}\left(\omega_{10}^{*}\right)^{3}\right) \sin 2 \tau_{2} \omega_{10}^{*} \\
& \left.-s_{1}\left(\omega_{10}^{*}\right)^{2} \cos 2 \tau_{2} \omega_{10}^{*}\right) \cos \tau_{10}^{*} \omega_{10}^{*} \text {, } \\
& Q_{4 I}=\left(n_{4}\left(\omega_{10}^{*}\right)^{5}-n_{2}\left(\omega_{10}^{*}\right)^{3}+n_{0} \omega_{10}^{*}\right. \\
& +\left(r_{0} \omega_{10}^{*}-r_{2}\left(\omega_{10}^{*}\right)^{3}\right) \cos \tau_{2} \omega_{10}^{*} \\
& -\left(r_{3}\left(\omega_{10}^{*}\right)^{4}-r_{1}\left(\omega_{10}^{*}\right)^{2}\right) \sin \tau_{2} \omega_{10}^{*} \\
& +\left(s_{0} \omega_{10}^{*}-s_{2}\left(\omega_{10}^{*}\right)^{3}\right) \cos 2 \tau_{2} \omega_{10}^{*} \\
& \left.+s_{1}\left(\omega_{10}^{*}\right)^{2} \sin 2 \tau_{2} \omega_{10}^{*}\right) \cos \tau_{10}^{*} \omega_{10}^{*} \\
& -\left(n_{3}\left(\omega_{10}^{*}\right)^{4}-n_{1}\left(\omega_{10}^{*}\right)^{2}+\left(r_{0} \omega_{10}^{*}-r_{2}\left(\omega_{10}^{*}\right)^{3}\right) \sin \tau_{2} \omega_{10}^{*}\right. \\
& +\left(r_{3}\left(\omega_{10}^{*}\right)^{4}-r_{1}\left(\omega_{10}^{*}\right)^{2}\right) \cos \tau_{2} \omega_{10}^{*} \\
& +\left(s_{0} \omega_{10}^{*}-s_{2}\left(\omega_{10}^{*}\right)^{3}\right) \sin 2 \tau_{2} \omega_{10}^{*} \\
& \left.-s_{1}\left(\omega_{10}^{*}\right)^{2} \cos 2 \tau_{2} \omega_{10}^{*}\right) \sin \tau_{10}^{*} \text {. }
\end{aligned}
$$


Obviously, if the condition $\left(H_{42}\right) P_{4 R} Q_{4 R}+P_{4 I} Q_{4 I} \neq 0$ holds, then $\operatorname{Re}\left[d \lambda / d \tau_{1}\right]_{\lambda=i \omega_{10}^{*}}^{-1} \neq 0$. According to the Hopf bifurcation theorem in [19], we have the following results for system (3).

Theorem 3. For system (3), if the conditions $\left(H_{41}\right)-\left(H_{42}\right)$ hold and $\tau_{2} \in\left(0, \tau_{20}\right)$, then the positive equilibrium $P^{*}\left(S^{*}, E^{*}, I^{*}, Q^{*}, R^{*}\right)$ of system (3) is asymptotically stable for $\tau_{1} \in\left[0, \tau_{10}^{*}\right)$ and system (3) undergoes a Hopf bifurcation at the positive equilibrium $P^{*}\left(S^{*}, E^{*}, I^{*}, Q^{*}, R^{*}\right)$ when $\tau_{1}=\tau_{10}^{*}$.

\section{Direction and Stability of the Hopf Bifurcation}

In this section, we determine the properties of the Hopf bifurcation of system (3) with respect to $\tau_{1}$ for $\tau_{2} \in\left(0, \tau_{20}\right)$. Throughout this section, we assume that $\tau_{20}^{*}<\tau_{10}^{*}$, where $\tau_{20}^{*} \in\left(0, \tau_{20}\right)$.

Let $\tau_{1}=\tau_{10}^{*}+\mu, \mu \in R$ so that $\mu=0$ is the Hopf bifurcation value of system (3). Rescaling the time delay by $t \rightarrow\left(t / \tau_{1}\right)$. Let $u_{1}(t)=S(t)-S^{*}, u_{2}(t)=E(t)-E^{*}, u_{3}(t)=I(t)-I^{*}$, $u_{4}(t)=Q(t)-Q^{*}, u_{5}(t)=R(t)-R^{*}$, then system (3) can be written as a PDE in $C=C\left([-1,0], R^{5}\right)$ :

$$
\dot{u}(t)=L_{\mu} u_{t}+F\left(\mu, u_{t}\right),
$$

and $L_{\mu}: C \rightarrow R^{5}, F: R \times C \rightarrow R^{5}$ are given, respectively, by

$$
\begin{gathered}
L_{\mu} \phi=\left(\tau_{10}^{*}+\mu\right)\left(A^{\prime} \phi(0)+C^{\prime}\left(-\frac{\tau_{20}^{*}}{\tau_{10}^{*}}\right)+B^{\prime} \phi(-1)\right), \\
F(\mu, \phi)=\left(\tau_{10}^{*}+\mu\right)\left(\begin{array}{c}
-\beta \phi_{1}(0) \phi_{3}(0) \\
\beta \phi_{1}(0) \phi_{3}(0) \\
0 \\
0 \\
0
\end{array}\right),
\end{gathered}
$$

with

$$
\begin{aligned}
A^{\prime} & =\left(\begin{array}{ccccc}
a_{11} & 0 & a_{13} & 0 & 0 \\
a_{21} & a_{22} & a_{23} & 0 & 0 \\
0 & a_{32} & 0 & 0 & 0 \\
0 & 0 & a_{43} & a_{44} & 0 \\
0 & 0 & 0 & 0 & a_{55}
\end{array}\right), \\
B^{\prime} & =\left(\begin{array}{ccccc}
0 & 0 & 0 & 0 & b_{15} \\
0 & 0 & 0 & 0 & 0 \\
0 & 0 & 0 & 0 & 0 \\
0 & 0 & 0 & 0 & 0 \\
0 & 0 & 0 & 0 & b_{55}
\end{array}\right) \\
C^{\prime} & =\left(\begin{array}{ccccc}
0 & 0 & 0 & 0 & 0 \\
0 & 0 & 0 & 0 & 0 \\
0 & 0 & c_{33} & 0 & 0 \\
0 & 0 & 0 & c_{44} & 0 \\
0 & 0 & c_{53} & c_{54} & 0
\end{array}\right)
\end{aligned}
$$

By the Riesz representation theorem, there exists a $5 \times 5$ matrix function $\eta(\theta, \mu):[-1,0] \rightarrow R^{5}$ whose elements are of bounded variation such that

$$
L_{\mu} \phi=\int_{-1}^{0} d \eta(\theta, \mu) \phi(\theta), \quad \phi \in C
$$

In fact, we choose

$$
\eta(\theta, \mu)= \begin{cases}\left(\tau_{10}^{*}+\mu\right)\left(A^{\prime}+B^{\prime}+C^{\prime}\right), & \theta=0, \\ \left(\tau_{10}^{*}+\mu\right)\left(B^{\prime}+C^{\prime}\right), & \theta \in\left[-\frac{\tau_{20}^{*}}{\tau_{10}^{*}}, 0\right), \\ \left(\tau_{10}^{*}+\mu\right) B^{\prime}, & \theta \in\left(-1,-\frac{\tau_{20}^{*}}{\tau_{10}^{*}}\right), \\ 0, & \theta=-1 .\end{cases}
$$

For $\phi \in C\left([-1,0], R^{5}\right)$, we define

$$
\begin{gathered}
A(\mu) \phi= \begin{cases}\frac{d \phi(\theta)}{d \theta}, & -1 \leq \theta<0, \\
\int_{-1}^{0} d \eta(\theta, \mu) \phi(\theta), & \theta=0,\end{cases} \\
R(\mu) \phi= \begin{cases}0, & -1 \leq \theta<0, \\
F(\mu, \phi), & \theta=0 .\end{cases}
\end{gathered}
$$

Then system (46) can be transformed into the following operator equation

$$
\dot{u}(t)=A(\mu) u_{t}+R(\mu) u_{t} .
$$

For $\varphi \in C\left([-1,0],\left(R^{5}\right)^{*}\right)$, we define the adjoint operator $A^{*}$ of $A$

$$
A^{*}(\varphi)= \begin{cases}-\frac{d \varphi(s)}{d s}, & 0<s \leq 1, \\ \int_{-1}^{0} d \eta^{T}(s, 0) \varphi(-s), & s=0,\end{cases}
$$

associated with a bilinear form

$$
\begin{aligned}
\langle\varphi(s), \phi(\theta)\rangle= & \bar{\varphi}(0) \phi(0) \\
& -\int_{\theta=-1}^{0} \int_{\xi=0}^{\theta} \bar{\varphi}(\xi-\theta) d \eta(\theta) \phi(\xi) d \xi,
\end{aligned}
$$

where $\eta(\theta)=\eta(\theta, 0)$. 
Let $\rho(\theta)=\left(1, \rho_{2}, \rho_{3}, \rho_{4}, \rho_{5}\right)^{T} e^{i \omega_{10}^{*} \tau_{10}^{*} \theta}$ be the eigenvector of $A$ corresponding to $+i \omega_{10}^{*} \tau_{10}^{*}$ and let $\rho^{*}(s)=D\left(1, \rho_{2}^{*}\right.$, $\left.\rho_{3}^{*}, \rho_{4}^{*}, \rho_{5}^{*}\right) e^{i \omega_{10}^{*} \tau_{10}^{*} s}$ be the eigenvector of $A^{*}$ corresponding to $-i \omega_{10}^{*} \tau_{10}^{*}$. From the definition of $A(0)$ and $A^{*}(0)$ and by a simple computation, we obtain

$$
\begin{gathered}
\rho_{2}=\frac{a_{21}+a_{23} \rho_{3}}{i \omega_{10}^{*}-a_{22}}, \\
\rho_{3}=\frac{a_{21} a_{32}}{\left(i \omega_{10}^{*}-a_{22}\right)\left(i \omega_{10}^{*}-c_{33} e^{-i \omega_{10}^{*} \tau_{20}^{*}}\right)-a_{23} a_{32}}, \\
\rho_{4}=\frac{a_{43} \rho_{3}}{i \omega_{10}^{*}-a_{44}-c_{44} e^{-i \omega_{10}^{*} \tau_{20}^{*}},} \\
\rho_{5}=\frac{i \omega_{10}^{*}-a_{11}-a_{13} \rho_{3}}{b_{15} e^{-i \omega_{10}^{*} \tau_{10}^{*}}}, \\
\left.\rho_{2}^{*}=-\frac{i \omega_{10}^{*}+a_{11}}{a_{21}}, \quad \rho_{3}^{*}=-\frac{\left(i \omega_{10}^{*}\right.}{a_{32}} a_{22}\right) \rho_{2}^{*} \\
\rho_{4}^{*}=-\frac{c_{54} e^{i \omega_{10}^{*} \tau_{20}^{*}} \rho_{5}^{*}}{i \omega_{10}^{*}+a_{44}+c_{44} e^{i \omega_{10}^{*} \tau_{20}^{*}}}, \\
\rho_{5}^{*}=-\frac{b_{15} e^{i \omega_{10}^{*} \tau_{10}^{*}}}{i \omega_{10}^{*}+a_{55}+b_{55} e^{i \omega_{10}^{*} \tau_{10}^{*}} .}
\end{gathered}
$$

From (54), we have

$$
\begin{aligned}
& \left\langle\rho^{*}(s), \rho(\theta)\right\rangle \\
& =\bar{D}\left[1+\rho_{2} \bar{\rho}_{2}^{*}+\rho_{3} \bar{\rho}_{3}^{*}+\rho_{4} \bar{\rho}_{4}^{*}+\rho_{5} \bar{\rho}_{5}^{*}+\tau_{10}^{*} e^{-i \omega_{10}^{*} \tau_{10}^{*}} \bar{\rho}_{5}^{*}\right. \\
& \quad+\tau_{20}^{*} e^{-i \omega_{10}^{*} \tau_{20}^{*}} \\
& \left.\quad \times\left(\bar{\rho}_{3}^{*}\left(c_{33} \rho_{3}+c_{53} \rho_{5}\right)+\bar{\rho}_{4}^{*}\left(c_{44} \rho_{4}+c_{54} \rho_{5}\right)\right)\right] .
\end{aligned}
$$

Let

$$
\begin{aligned}
\bar{D}= & {\left[1+\rho_{2} \bar{\rho}_{2}^{*}+\rho_{3} \bar{\rho}_{3}^{*}+\rho_{4} \bar{\rho}_{4}^{*}+\rho_{5} \bar{\rho}_{5}^{*}\right.} \\
& +\tau_{10}^{*} e^{-i \omega_{10}^{*} \tau_{10}^{*}} \bar{\rho}_{5}^{*}+\tau_{20}^{*} e^{-i \omega_{10}^{*} \tau_{20}^{*}} \\
& \left.\times\left(\bar{\rho}_{3}^{*}\left(c_{33} \rho_{3}+c_{53} \rho_{5}\right)+\bar{\rho}_{4}^{*}\left(c_{44} \rho_{4}+c_{54} \rho_{5}\right)\right)\right]^{-1} .
\end{aligned}
$$

Then, $\left\langle\rho^{*}, \rho\right\rangle=1,\left\langle\rho^{*}, \bar{\rho}\right\rangle=0$.

Next, we can obtain the coefficients determining the properties of the Hopf bifurcation by the algorithms introduced in [19] and using a computation process similar to that in $[20]$ :

$$
\begin{aligned}
g_{20}= & 2 \beta \tau_{10}^{*} \bar{D}\left(\bar{\rho}_{2}^{*}-1\right) \rho^{(1)}(0) \rho^{(3)}(0), \\
g_{11}= & \beta \tau_{10}^{*} \bar{D}\left(\bar{\rho}_{2}^{*}-1\right)\left(\bar{\rho}^{(1)}(0) \rho^{(3)}(0)+\rho^{(1)}(0) \bar{\rho}^{(3)}(0)\right), \\
g_{02}= & 2 \beta \tau_{10}^{*} \bar{D}\left(\bar{\rho}_{2}^{*}-1\right) \bar{\rho}^{(1)}(0) \bar{\rho}^{(3)}(0), \\
g_{21}= & 2 \beta \tau_{10}^{*} \bar{D}\left(\bar{\rho}_{2}^{*}-1\right) \\
& \times\left(W_{11}^{(1)}(0) \rho^{(3)}(0)+\frac{1}{2} W_{20}^{(1)}(0) \bar{\rho}^{(3)}(0)\right. \\
& \left.\quad+W_{11}^{(3)}(0) \rho^{(1)}(0)+\frac{1}{2} W_{20}^{(3)}(0) \bar{\rho}^{(1)}(0)\right),
\end{aligned}
$$

with

$$
\begin{gathered}
W_{20}(\theta)=\frac{i g_{20} \rho(0)}{\tau_{10}^{*} \omega_{10}^{*}} e^{i \tau_{10}^{*} \omega_{10}^{*} \theta}+\frac{i \bar{g}_{02} \bar{\rho}(0)}{3 \tau_{10}^{*} \omega_{10}^{*}} e^{-i \tau_{10}^{*} \omega_{10}^{*} \theta} \\
+E_{1} e^{2 i \tau_{10}^{*} \omega_{10}^{*} \theta}, \\
W_{11}(\theta)=-\frac{i g_{11} \rho(0)}{\tau_{10}^{*} \omega_{10}^{*}} e^{i \tau_{10}^{*} \omega_{10}^{*}}+\frac{i \bar{g}_{11} \bar{\rho}(0)}{\tau_{10}^{*} \omega_{10}^{*}} e^{-i \tau_{10}^{*} \omega_{10}^{*} \theta}+E_{2},
\end{gathered}
$$

where $E_{1}$ and $E_{2}$ can be determined by the following equations, respectively:

$$
\begin{gathered}
E_{1}=2\left(\begin{array}{ccccc}
a_{11}^{\prime} & 0 & -a_{13} & 0 & -b_{15} e^{-2 i \omega_{10}^{*} \tau_{10}^{*}} \\
-a_{21} & a_{22}^{\prime} & -a_{23} & 0 & 0 \\
0 & -a_{32} & a_{33}^{\prime} & 0 & 0 \\
0 & 0 & -a_{43} & a_{44}^{\prime} & 0 \\
0 & 0 & -c_{53} e^{-2 i \omega_{10}^{*} \tau_{20}^{*}} & -c_{54} e^{-2 i \omega_{10}^{*} \tau_{20}^{*}} & a_{55}^{\prime}
\end{array}\right)^{-1}\left(\begin{array}{c}
E_{1}^{(1)} \\
E_{1}^{(2)} \\
0 \\
0 \\
0
\end{array}\right), \\
E_{2}=-\left(\begin{array}{ccccc}
a_{11} & 0 & a_{13} & 0 & b_{15} \\
a_{21} & a_{22} & a_{23} & 0 & 0 \\
0 & a_{32} & c_{33} & 0 & 0 \\
0 & 0 & a_{43} & a_{44}+c_{44} & 0 \\
0 & 0 & c_{53} & c_{54} & a_{55}+b_{55}
\end{array}\right)^{-1}\left(\begin{array}{c}
E_{2}^{(1)} \\
E_{2}^{(2)} \\
0 \\
0 \\
0
\end{array}\right),
\end{gathered}
$$




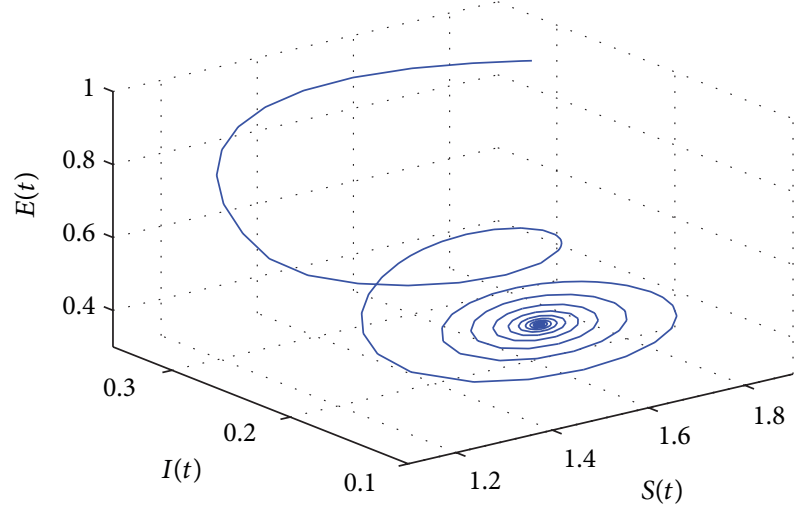

FIgURE 1: The phase plot of the states $S, E$, and $I$ for $\tau_{1}=7.85<$ $8.4755=\tau_{10}$.

with

$$
\begin{aligned}
& a_{11}^{\prime}=2 i \omega_{10}^{*}-a_{11}, \\
& a_{22}^{\prime}=2 i \omega_{10}^{*}-a_{22}, \\
& a_{33}^{\prime}=2 i \omega_{10}^{*}-c_{33} e^{-2 i \omega_{10}^{*} \tau_{20}^{*},} \\
& a_{44}^{\prime}=2 i \omega_{10}^{*}-a_{44}-c_{44} e^{-2 i \omega_{10}^{*} \tau_{20}^{*},} \\
& a_{55}^{\prime}=2 i \omega_{10}^{*}-a_{55}-b_{55} e^{-2 i \omega_{10}^{*} \tau_{10}^{*},} \\
& E_{1}^{(1)}=-\beta \rho^{(1)}(0) \rho^{(3)}(0), \\
& E_{1}^{(2)}=\beta \rho^{(1)}(0) \rho^{(3)}(0), \\
& E_{2}^{(1)}=-\beta\left(\bar{\rho}^{(1)}(0) \rho^{(3)}(0)+\rho^{(1)}(0) \bar{\rho}^{(3)}(0)\right), \\
& E_{2}^{(2)}=\beta\left(\bar{\rho}^{(1)}(0) \rho^{(3)}(0)+\rho^{(1)}(0) \bar{\rho}^{(3)}(0)\right) .
\end{aligned}
$$

Then, we can get the following coefficients:

$$
\begin{aligned}
& C_{1}(0)=\frac{i}{2 \tau_{10}^{*} \omega_{10}^{*}}\left(g_{11} g_{20}-2\left|g_{11}\right|^{2}-\frac{\left|g_{02}\right|^{2}}{3}\right)+\frac{g_{21}}{2}, \\
& \mu_{2}=-\frac{\operatorname{Re}\left\{C_{1}(0)\right\}}{\operatorname{Re}\left\{\lambda^{\prime}\left(\tau_{10}^{*}\right)\right\}} \\
& \beta_{2}=2 \operatorname{Re}\left\{C_{1}(0)\right\} \\
& T_{2}=-\frac{\operatorname{Im}\left\{C_{1}(0)\right\}+\mu_{2} \operatorname{Im}\left\{\lambda^{\prime}\left(\tau_{10}^{*}\right)\right\}}{\tau_{10}^{*} \omega_{10}^{*}} .
\end{aligned}
$$

In conclusion, we have the following results.

Theorem 4. For system (3), if $\mu_{2}>0\left(\mu_{2}<0\right)$, the Hopf bifurcation is supercritical (subcritical). If $\beta_{2}<0\left(\beta_{2}>0\right)$ the bifurcating periodic solutions are stable (unstable). If $T_{2}>$ $0\left(T_{2}<0\right)$, the period of the bifurcating periodic solutions increases (decreases).

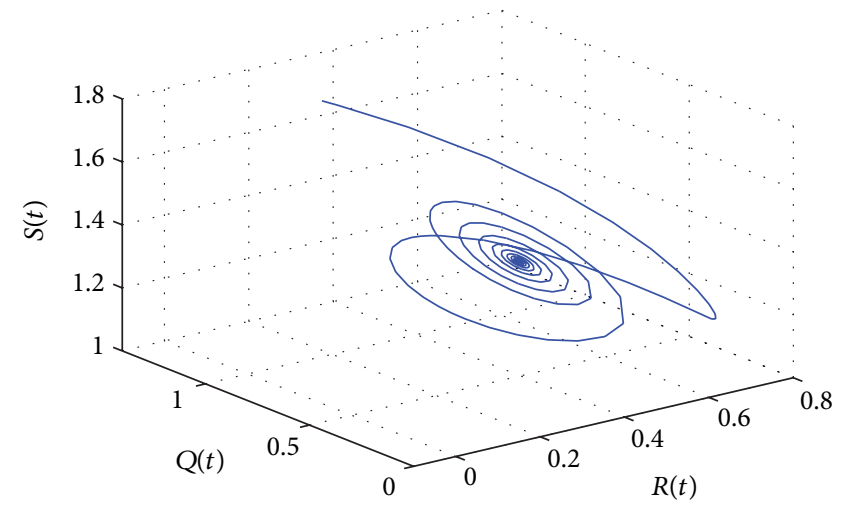

Figure 2: The phase plot of the states $S, Q$, and $R$ for $\tau_{1}=7.85<$ $8.4755=\tau_{10}$.

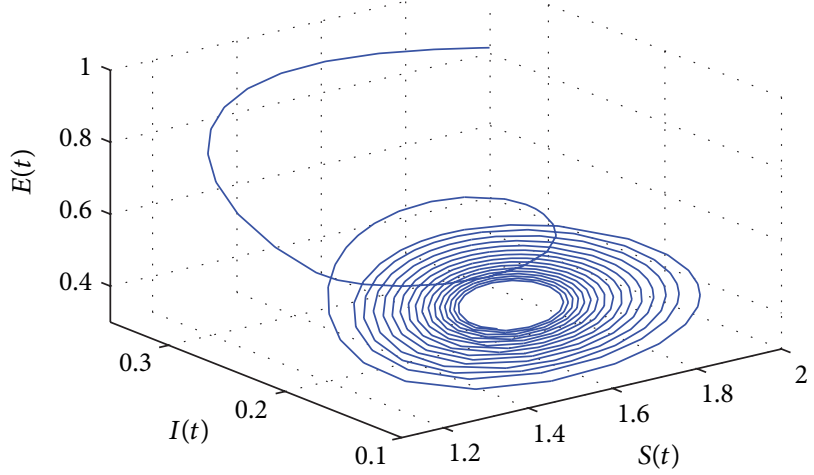

Figure 3: The phase plot of the states $S, E$, and $I$ for $\tau_{1}=9.85>$ $8.4755=\tau_{10}$.

\section{Numerical Simulation}

In this section, we present some numerical simulations to verify the theoretical results in Sections 2 and 3. Let $A=0.33$, $\beta=0.75, d=0.1, \eta=0.2, \mu=0.3, \alpha=0.2, \gamma=0.18, \delta=0.38$, $\varepsilon=0.3$. Then, we get the following particular case of system (3):

$$
\begin{aligned}
\frac{d S(t)}{d t}= & 0.33-0.75 S(t) I(t)-0.1 S(t)+0.2 R\left(t-\tau_{1}\right), \\
\frac{d E(t)}{d t}= & 0.75 S(t) I(t)-0.4 E(t), \\
\frac{d I(t)}{d t}= & 0.3 E(t)-0.68 I(t)-0.18 I\left(t-\tau_{2}\right), \\
\frac{d Q(t)}{d t}= & 0.38 I(t)-0.3 Q(t)-0.3 Q\left(t-\tau_{2}\right), \\
\frac{d R(t)}{d t}= & 0.18 I\left(t-\tau_{2}\right)+0.3 Q\left(t-\tau_{2}\right)-0.1 R(t), \\
& -0.2 R\left(t-\tau_{1}\right) .
\end{aligned}
$$




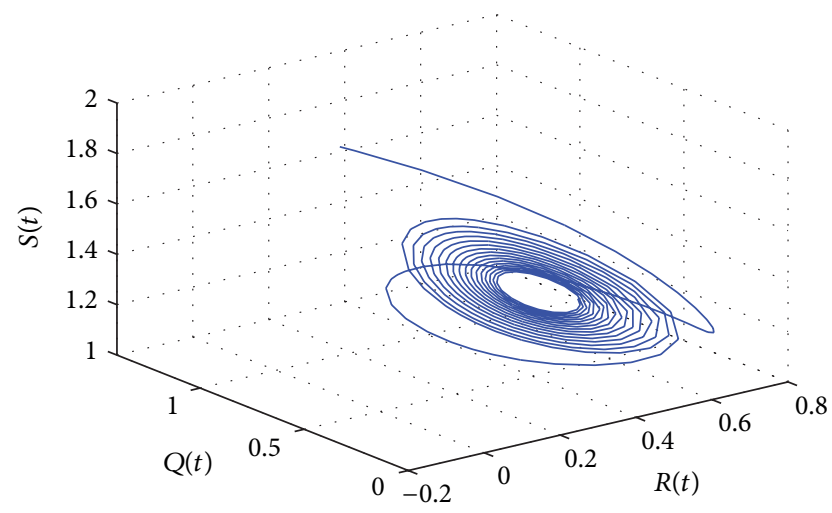

Figure 4: The phase plot of the states $S, Q$, and $R$ for $\tau_{1}=9.85>$ $8.4755=\tau_{10}$.

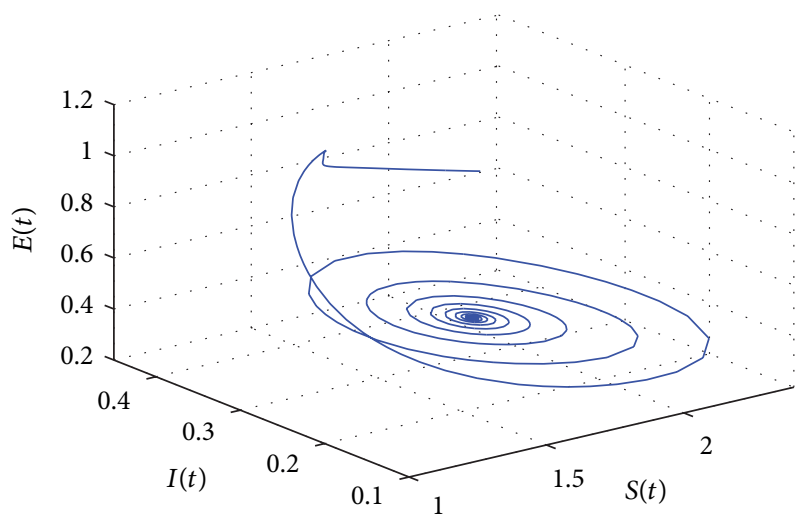

FIGURE 5: The phase plot of the states $S, E$, and $I$ for $\tau_{2}=7.75<$ $8.1081=\tau_{20}$.

It is easy to verify that $R_{0}=2.1584>1$. Then, we get the unique positive equilibrium $P^{*}(1.5289,0.5656,0.1973$, $0.1250,0.2433)$ of system (63). Further, we can obtain $\operatorname{det}_{1}=$ $2.4080>0, \operatorname{det}_{2}=3.7619>0, \operatorname{det}_{3}=2.2 .0074>0$, $\operatorname{det}_{4}=0.1012>0$, and $\operatorname{det}_{5}=0.0016>0$. That is, the condition $\left(H_{1}\right)$ holds.

For $\tau_{1}>0, \tau_{2}=0$. By some complex computation, we obtain $\omega_{10}=1.3397, \tau_{10}=8.4755$, and $f_{1}^{\prime}\left(v_{1 *}\right)=2.3102>$ 0 . That is, the conditions $\left(H_{21}\right)$ and $\left(H_{22}\right)$ hold. According to Theorem 1, we can conclude that when $\tau_{1} \in\left[0, \tau_{10}\right)$, the positive equilibrium $P^{*}(1.5289,0.5656,0.1973,0.1250$, 0.2433 ) of system (63) is asymptotically stable. However, when the value of $\tau_{1}$ passes through the critical value $\tau_{10}$, the positive equilibrium $P^{*}(1.5289,0.5656,0.1973,0.1250$, 0.2433 ) of system (63) will lose its stability and a Hopf bifurcation occurs at the positive equilibrium of system (63). This property can be illustrated by Figures $1-4$. As can be seen from Figures 1-2, if we choose $\tau_{1}=7.85<\tau_{10}$, it is easy to see from Figures 1-2 that the positive equilibrium $P^{*}(1.5289$, $0.5656,0.1973,0.1250,0.2433)$ of system (63) is asymptotically stable. However, if we choose $\tau_{1}=9.85>$ $\tau_{10}$, then the positive equilibrium $P^{*}(1.5289,0.5656,0.1973$, $0.1250,0.2433$ ) loses its stability and a Hopf bifurcation occurs, which can be illustrated by Figures 3-4. Similarly, we

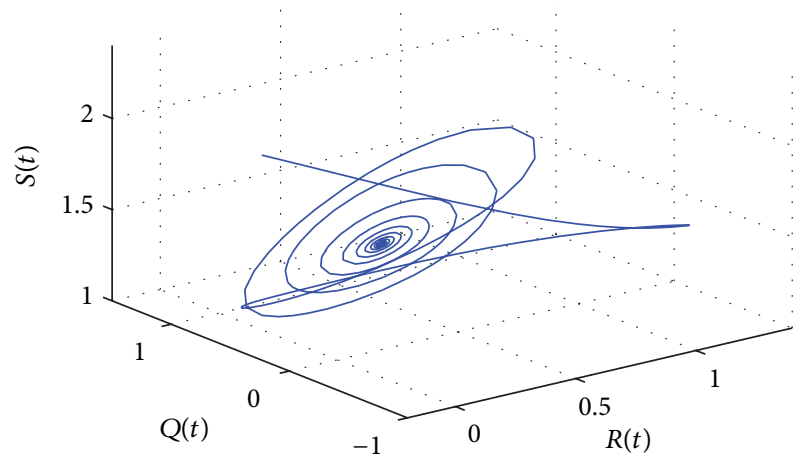

FIgURE 6: The phase plot of the states $S, Q$, and $R$ for $\tau_{2}=7.75<$ $8.1081=\tau_{20}$.

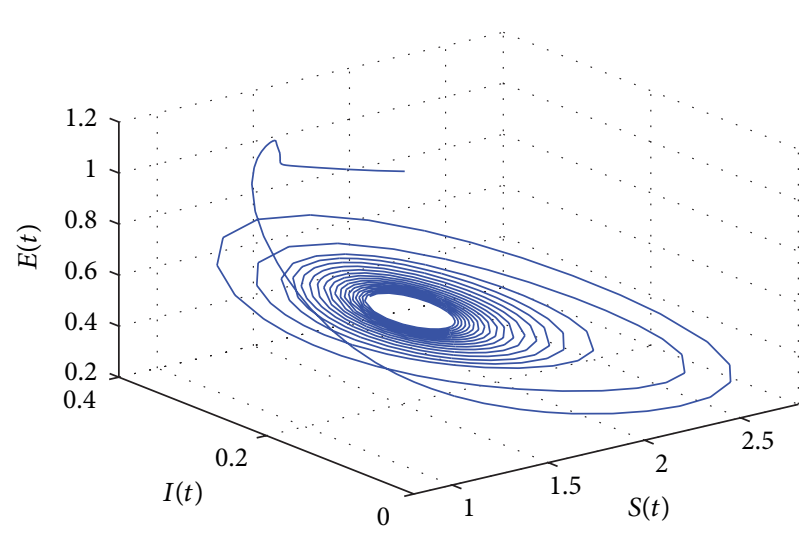

Figure 7: The phase plot of the states $S, E$, and $I$ for $\tau_{2}=9.37>$ $8.1081=\tau_{20}$.

have $\omega_{20}=1.7690, \tau_{20}=8.1081$ and $P_{3 R} Q_{3 R}+P_{3 I} Q_{3 I}=$ $0.0319>0$. Namely, the conditions $\left(H_{31}\right)$ and $\left(H_{32}\right)$ hold. The corresponding phase plots are shown in Figures 5, 6, 7, and 8.

For $\tau_{1}>0, \tau_{2}>0$ and $\tau_{2}=5.25 \in\left(0, \tau_{20}\right)$. We obtain $\omega_{10}^{*}=3.6529, \tau_{10}^{*}=5.6477$ by some complex computations. The corresponding phase plots are shown in Figures 9-12. As illustrated by Figures 9-10, when $\tau_{1}=$ $5.05 \in\left(0, \tau_{10}^{*}\right)$, the positive equilibrium $P^{*}(1.5289,0.5656$, $0.1973,0.1250,0.2433)$ of system $(63)$ is asymptotically stable. However, as can be seen from Figures 11-12, the positive equilibrium $P^{*}(1.5289,0.5656,0.1973,0.1250,0.2433)$ of system (63) becomes unstable and a Hopf bifurcation occurs at $P^{*}(1.5289,0.5656,0.1973,0.1250,0.2433)$ when $\tau_{1}=6.25>$ $\tau_{10}^{*}$. This property is consistent with Theorem 3 . In addition, we have $\lambda^{\prime}\left(\tau_{10}^{*}\right)=0.0493+0.0126 i, C_{1}(0)=-5.8133+2.5756 i$. Thus, we have $\mu_{2}=117.9168>0, \beta_{2}=-11.6266<0$, $T_{2}=-0.2711<0$. From Theorem 4 , we can conclude that the Hopf bifurcation is supercritical and the bifurcating periodic solutions are stable, and the period of the periodic solutions decreases. 


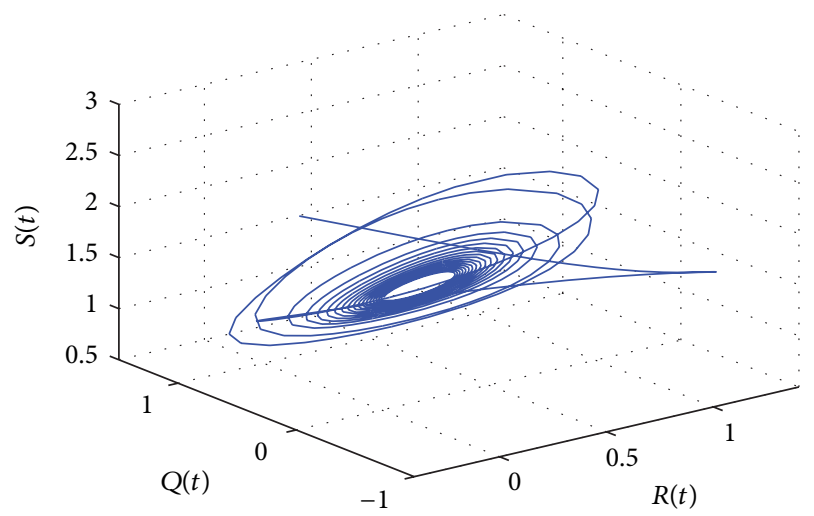

FIGURE 8: The phase plot of the states $S, Q$, and $R$ for $\tau_{2}=9.37>$ $8.1081=\tau_{20}$.

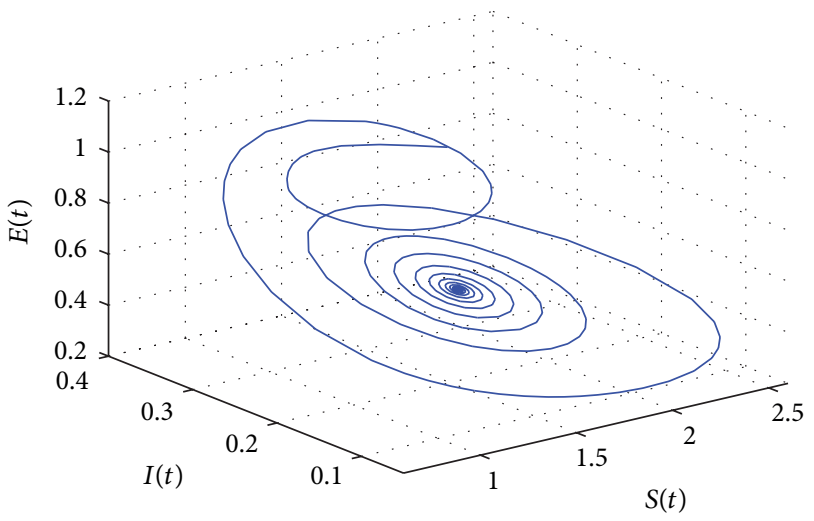

Figure 9: The phase plot of the states $S, E$, and $I$ for $\tau_{1}=5.05<$ $5.6477=\tau_{10}^{*}$ and $\tau_{2}=5.25 \in\left(0, \tau_{20}\right)$.

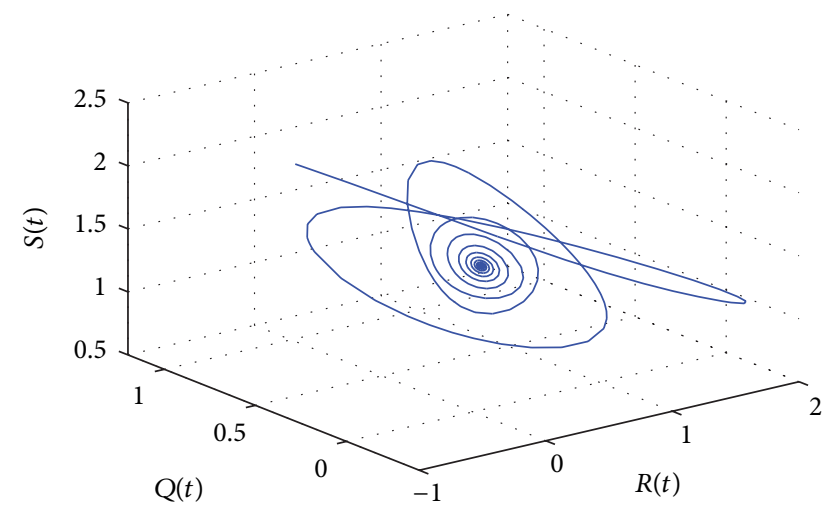

Figure 10: The phase plot of the states $S, Q$, and $R$ for $\tau_{1}=5.05<$ $5.6477=\tau_{10}^{*}$ and $\tau_{2}=5.25 \in\left(0, \tau_{20}\right)$.

\section{Conclusions}

This paper is concerned with a delayed SEIQRS model for the transmission of malicious objects in computer network. Compared with the literature [12], we consider not only the time delay due to the temporary immunity period but also the time delay due to the period that the infected computer

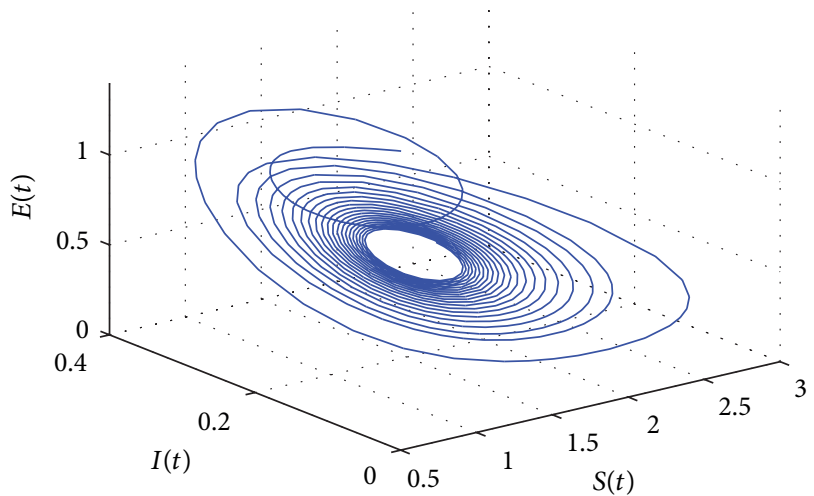

FIGURE 11: The phase plot of the states $S, E$, and $I$ for $\tau_{1}=6.25>$ $5.6477=\tau_{10}^{*}$ and $\tau_{2}=5.25 \in\left(0, \tau_{20}\right)$.

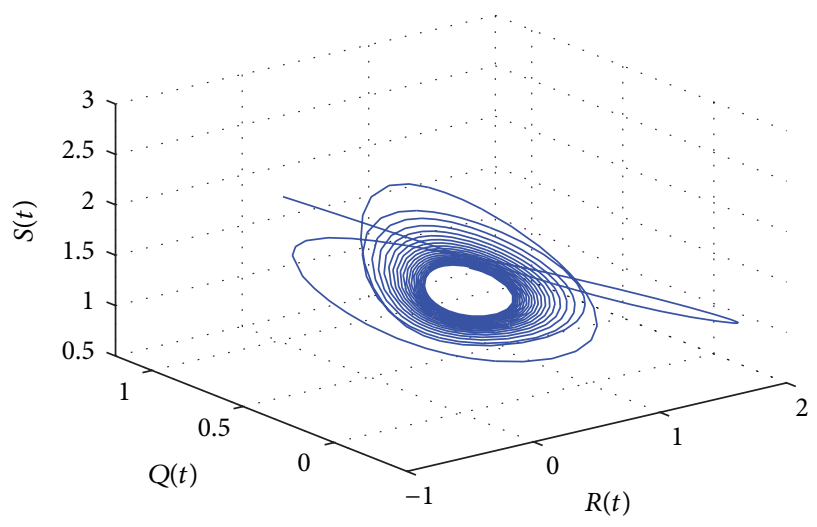

Figure 12: The phase plot of the states $S, Q$, and $R$ for $\tau_{1}=6.25>$ $5.6477=\tau_{10}^{*}$ and $\tau_{2}=5.25 \in\left(0, \tau_{20}\right)$.

uses to clean viruses by antivirus software. That is, the system we considered in this paper is more general than that in the literature [12]. By considering the possible combination of the two delays as a bifurcation parameter, we find that when the delay is below the corresponding critical value, the positive equilibrium of system (3) is locally asymptotically stable. However, when the delay passes through the corresponding critical value, the positive equilibrium of system (3) loses its stability and system (3) undergoes a Hopf bifurcation, which is not welcomed in networks. Furthermore, direction of the Hopf bifurcation and stability of the bifurcating periodic solutions are determined by using the normal form method and center manifold theory. Numerical simulations are presented to illustrate the theoretical analysis and results. Since the occurrence of the Hopf bifurcation is not welcomed in networks, we should control the Hopf bifurcation by some bifurcation control strategies such as the state feedback and parameter perturbation and so on. This is a further problem, which can be studied in the future.

\section{Conflict of Interests}

The authors declare that there is no conflict of interests regarding the publication of this paper. 


\section{Acknowledgments}

The authors are grateful to the anonymous referees and the editor for their valuable comments and suggestions on the paper. This work was supported by the National Naturall Science Foundation of China (61273070), a Project Funded by the Priority Academic Program Development of Jiangsu Higher Education Institutions and Natural Science Foundation of the Higher Education Institutions of Anhui Province (KJ2014A005).

\section{References}

[1] R. W. Thommes and M. J. Coates, "Modeling virus propagation in peer-to-peer networks," in Proceedings of the 5th International Conference on Information, Communications and Signal Processing, pp. 981-985, Bangkok, Thailand, December 2005.

[2] B. K. Mishra and D. K. Saini, "SEIRS epidemic model with delay for transmission of malicious objects in computer network," Applied Mathematics and Computation, vol. 188, no. 2, pp. 14761482, 2007.

[3] L. Wen and J. Zhong, "Global asymptotic stability and a property of the SIS model on bipartite networks," Nonlinear Analysis: Real World Applications, vol. 13, no. 2, pp. 967-976, 2012.

[4] B. K. Mishra and N. Jha, "SEIQRS model for the transmission of malicious objects in computer network," Applied Mathematical Modelling, vol. 34, no. 3, pp. 710-715, 2010.

[5] J. O. Kephart and S. R. White, "Directed-graph epidemiological models of computer viruses," in Proceedings of the IEEE Computer Society Symposium on Research in Security and Privacy, pp. 343-359, May 1991.

[6] J. G. Ren, X. F. Yang, L. X. Yang, Y. Xu, and F. Yang, "A delayed computer virus propagation model and its dynamics," Chaos, Solitons \& Fractals, vol. 45, no. 1, pp. 74-79, 2012.

[7] L. Feng, X. Liao, H. Li, and Q. Han, "Hopf bifurcation analysis of a delayed viral infection model in computer networks," Mathematical and Computer Modelling, vol. 56, no. 7-8, pp. 167179, 2012.

[8] T. Dong, X. Liao, and H. Li, "Stability and Hopf bifurcation in a computer virus model with multistate antivirus," Abstract and Applied Analysis, vol. 2012, Article ID 841987, 16 pages, 2012.

[9] Z. Zhang and H. Yang, "Stability and Hopf bifurcation in a delayed SEIRS worm model in computer network," Mathematical Problems in Engineering, vol. 2013, Article ID 319174, 9 pages, 2013.

[10] Y. Yao, W. Xiang, A. Qu, G. Yu, and F. Gao, "Hopf bifurcation in an SEIDQV worm propagation model with quarantine strategy," Discrete Dynamics in Nature and Society, vol. 2012, Article ID 304868, 18 pages, 2012.

[11] Y. Yao, N. Zhang, W. L. Xiang, G. Yu, and F. X. Gao, "Modeling and analysis of bifurcation in a delayed worm propagation model," Journal of Applied Mathematics, vol. 2013, Article ID 927369, 11 pages, 2013.

[12] J. Liu, "Hopf bifurcation in a delayed SEIQRS model for the transmission of malicious objects in computer network," Journal of Applied Mathematics, vol. 2014, Article ID 492198, 8 pages, 2014.

[13] C. J. Xu and X. F. He, "Stability and bifurcation analysis in a class of two-neuron networks with resonant bilinear terms," Abstract and Applied Analysis, vol. 2011, Article ID 697630, 21 pages, 2011.
[14] S. Gakkhar and A. Singh, "Complex dynamics in a prey predator system with multiple delays," Communications in Nonlinear Science and Numerical Simulation, vol. 17, no. 2, pp. 914-929, 2012.

[15] J. Liu, C. W. Sun, and Y. M. Li, "Stability and Hopf bifurcation analysis for a Gause-type predator-prey system with multiple delays," Abstract and Applied Analysis, vol. 2013, Article ID 795358, 12 pages, 2013.

[16] X. Meng, H. Huo, and H. Xiang, "Hopf bifurcation in a threespecies system with delays," Journal of Applied Mathematics and Computing, vol. 35, no. 1-2, pp. 635-661, 2011.

[17] Z. Zhang, H. Yang, and J. Liu, "Stability and Hopf bifurcation in a modified HOLling-Tanner predator-prey system with multiple delays," Abstract and Applied Analysis, vol. 2012, Article ID 236484, 19 pages, 2012.

[18] X. Meng, H. Huo, X. B. Zhang, and H. Xiang, "Stability and Hopf bifurcation in a three-species system with feedback delays," Nonlinear Dynamics, vol. 64, no. 4, pp. 349-364, 2011.

[19] B. D. Hassard, N. D. Kazarinoff, and Y. H. Wan, Theory and Applications of Hopf Bifurcation, Cambridge University Press, Cambridge, UK, 1981.

[20] C. Bianca, M. Ferrara, and L. Guerrini, “The Cai model with time delay: existence of periodic solutions and asymptotic analysis," Applied Mathematics \& Information Sciences, vol. 7, no. 1, pp. 21-27, 2013. 


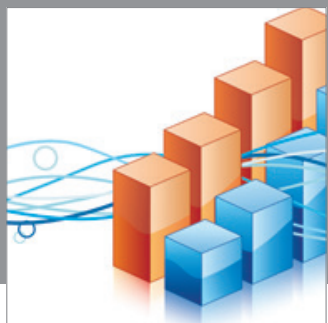

Advances in

Operations Research

mansans

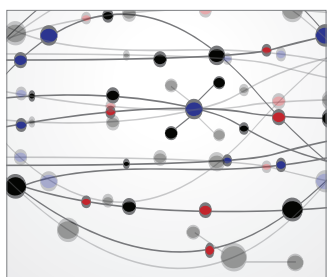

The Scientific World Journal
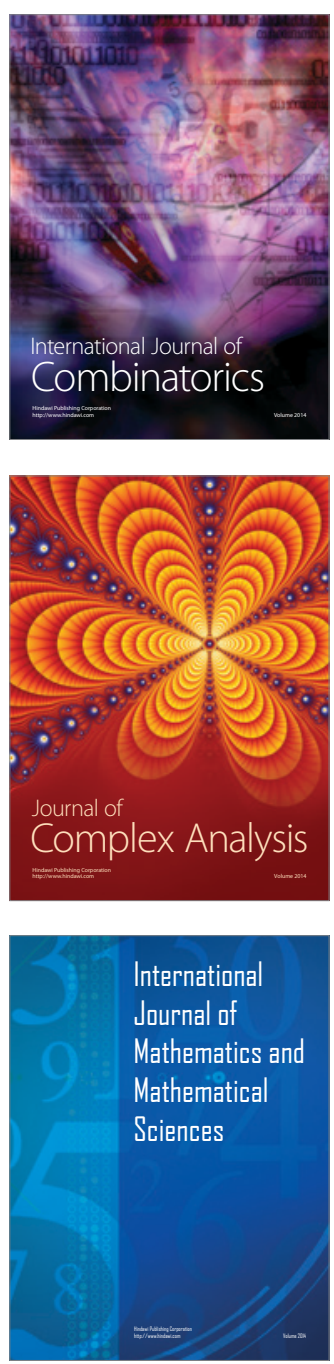
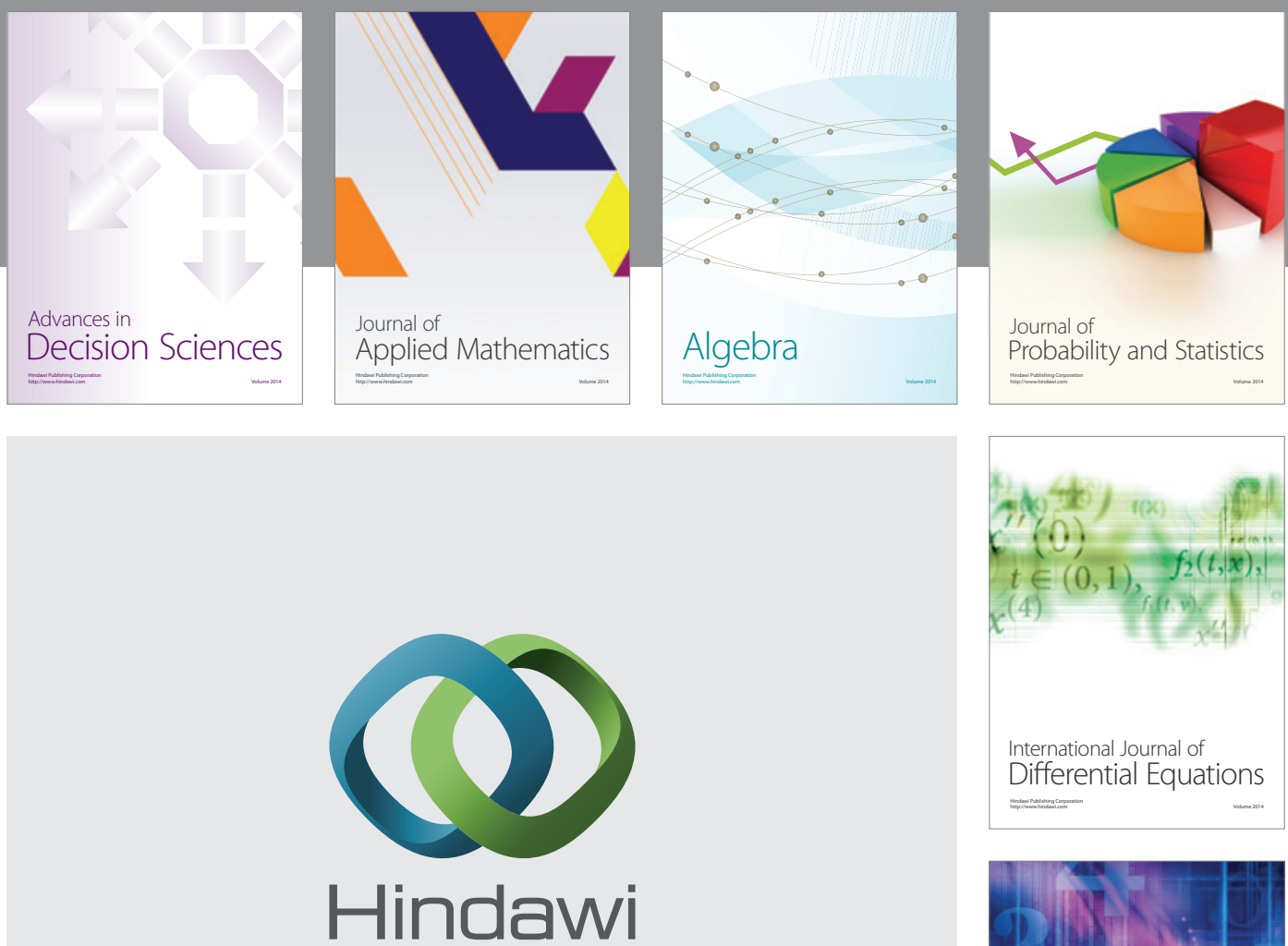

Submit your manuscripts at http://www.hindawi.com
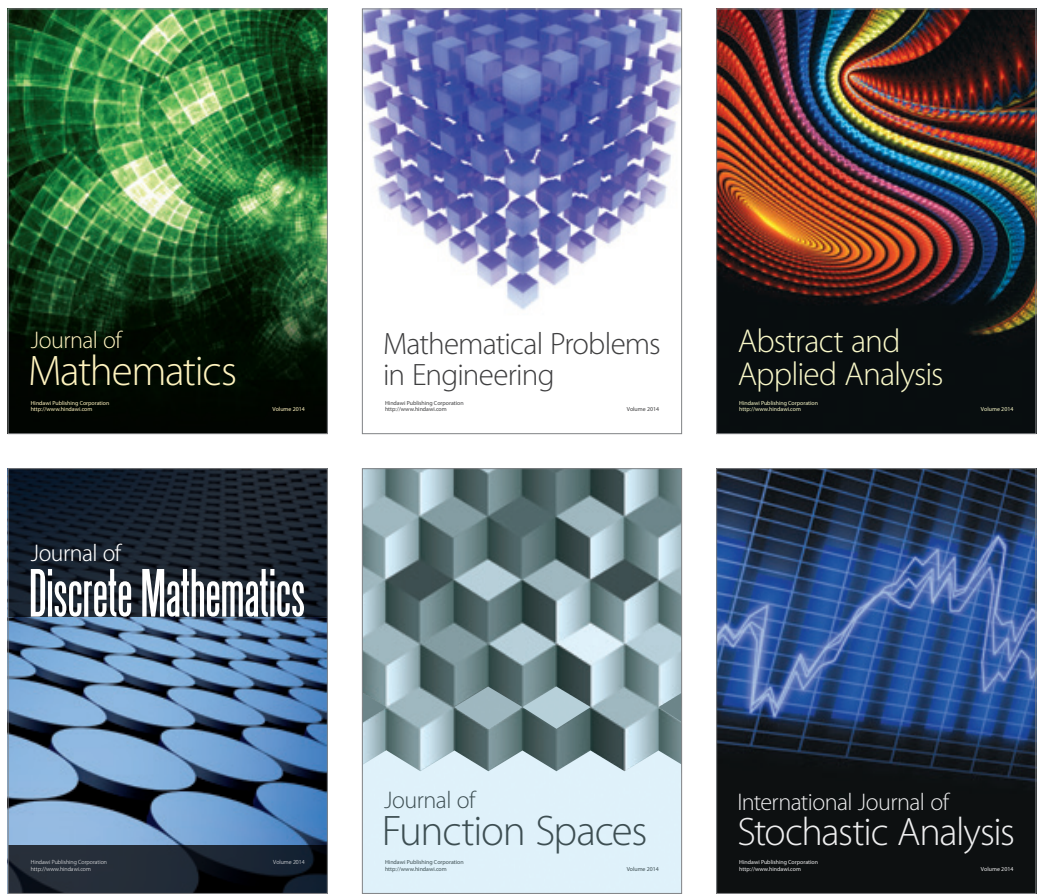

Journal of

Function Spaces

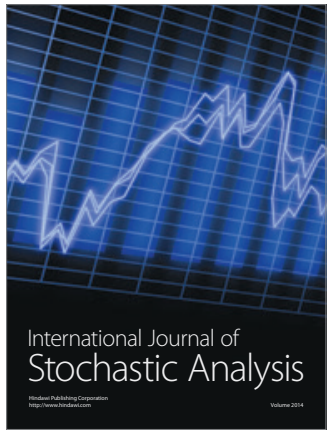

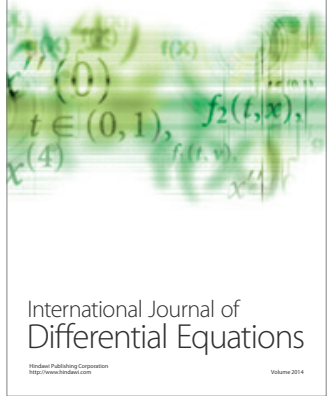
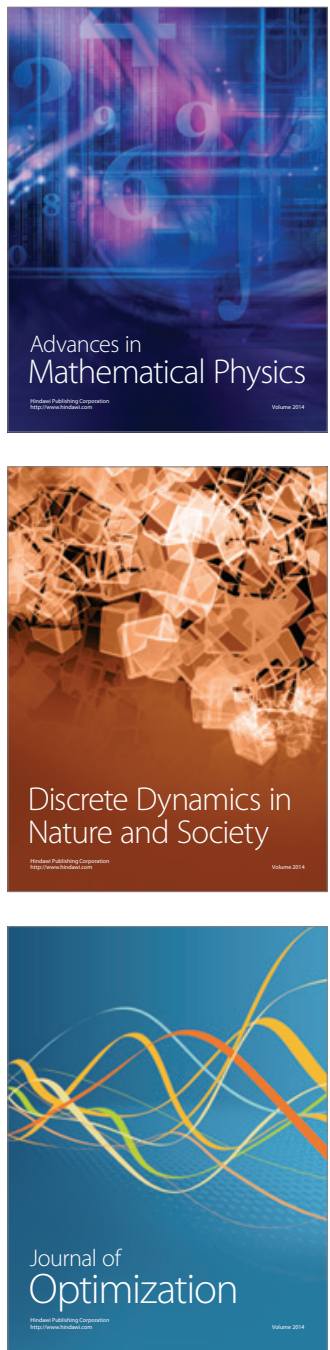